\title{
Introductory overview of identifiability analysis: A guide to evaluating whether you have the right type of data for your modeling purpose
}

\author{
Joseph H.A. Guillaume ${ }^{\mathrm{a}, \mathrm{b}, *}$, John D. Jakeman ${ }^{\mathrm{c}}$, Stefano Marsili-Libelli ${ }^{\mathrm{d}}$, Michael Asher ${ }^{\mathrm{b}}$, \\ Philip Brunner ${ }^{\mathrm{e}}$, Barry Croke ${ }^{\mathrm{b}}$, Mary C. Hill ${ }^{\mathrm{f}}$, Anthony J. Jakeman ${ }^{\mathrm{b}}$, Karel J. Keesman ${ }^{\mathrm{g}}$, \\ Saman Razavi ${ }^{\text {h, }}$ Johannes D. Stigter ${ }^{g}$ \\ ${ }^{a}$ Water and Development Research Group, Aalto University, Finland \\ ${ }^{\mathrm{b}}$ Fenner School of Environment \& Society, Australian National University, Australia \\ ${ }^{\mathrm{c}}$ Optimization and Uncertainty Quantification, Sandia National Laboratories, USA \\ ${ }^{\mathrm{d}}$ Department of Information Engineering, School of Engineering, University of Florence, Italy \\ ${ }^{\text {e } C e n t r e ~ d ' H y d r o g e ́ o l o g i e ~ e t ~ d e ~ G e ́ o t h e r m i e ~(C H Y N), ~ U n i v e r s i t e ́ ~ d e ~ N e u c h a ̂ t e l, ~ S w i t z e r l a n d ~}$ \\ ${ }^{\mathrm{f}}$ University of Kansas, USA \\ ${ }^{\mathrm{g}}$ Mathematical and Statistical Methods Group, Wageningen University, the Netherlands \\ ${ }^{\mathrm{h}}$ Global Institute for Water Security, School of Environment and Sustainability, and Department of Civil, Geological, and Environmental Engineering, University of \\ Saskatchewan, Saskatoon, Saskatchewan, Canada
}

\section{A R T I C L E I N F O}

\section{Keywords:}

Identifiability

Response surface

Non-uniqueness

Derivative based methods

Hessian

Emulation

Uncertainty

\begin{abstract}
A B S T R A C T
Identifiability is a fundamental concept in parameter estimation, and therefore key to the large majority of environmental modeling applications. Parameter identifiability analysis assesses whether it is theoretically possible to estimate unique parameter values from data, given the quantities measured, conditions present in the forcing data, model structure (and objective function), and properties of errors in the model and observations. In other words, it tackles the problem of whether the right type of data is available to estimate the desired parameter values. Identifiability analysis is therefore an essential technique that should be adopted more routinely in practice, alongside complementary methods such as uncertainty analysis and evaluation of model performance. This article provides an introductory overview to the topic. We recommend that any modeling study should document whether a model is non-identifiable, the source of potential non-identifiability, and how this affects intended project outcomes.
\end{abstract}

Learning objectives

- Appreciate key concepts and methods of parameter identifiability analysis

- Recognise the main consequences of parameter non-identifiability, i.e., the inability to infer unique parameters from data

- Distinguish between different sources of parameter non-uniqueness and how they influence identifiability

- Understand that non-uniqueness can occur even with ideal and/or noise-free data

- Recognise non-identifiability can be due to model structure and equations alone

- Understand how non-uniqueness in parameter estimation may relate to multiple optima and/or flatness in the response surface

- Investigate potential multiple optima and flatness using visualization, derivatives and related indicators

- Understand that noise in data can affect identifiability

- Appreciate issues affecting use of identifiability analysis, including computational considerations

\section{Introduction: what is identifiability analysis?}

Models are widely used for understanding, management and scenario analysis of environmental (and other) systems, increasingly for social learning among stakeholders, and in support of decision making (Kelly (Letcher) et al., 2013). The ultimate aim is to utilize data and knowledge about a system to help the modeler, decision makers and other stakeholders make sense of how the system works, how it may change in future, and how it may respond to management actions and other perturbations. In an ideal world, data and knowledge about a

\footnotetext{
* Corresponding author. Water and Development Research Group, Aalto University, Finland.

E-mail address: joseph.guillaume@anu.edu.au (J.H.A. Guillaume).
} 


\section{How well has uncertainty been reduced?}

Parameter estimation

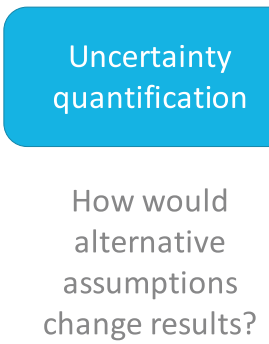

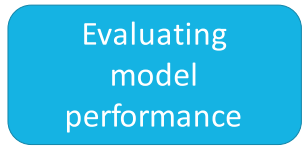

\author{
How well do \\ predictions match \\ observations?
}


What parameter
values fit the
observations?

Identifiability

analysis
Can the types of
observations used
yield unique
parametervalues?

Fig. 1. The role of identifiability analysis with respect to other methods.

system of interest would be sufficiently complete such that the model of that system would become an oracle that can be trusted to always provide the right answer.

In reality, we typically have incomplete knowledge and data to adequately conceptualise and simulate a system, and even in cases where we have an adequate understanding of the general principles governing the system, we can typically only approximate its complexity, spatial heterogeneity and temporal variability. Tackling this lack of "certainty" requires a pragmatic approach to managing information (i.e. data or knowledge that informs understanding of the system), underpinned by effective modeling practice (e.g. Badham et al., 2019), and driven by the purpose of the analysis and the resources available. It is necessary to balance efforts to: 1) obtain costeffective information, 2) make best use of that information to manage and reduce critical controls on uncertainty, and 3) understand the remaining uncertainty in order to recognise the limitations on how the model should be used.

To develop a model, there are several steps (e.g. Jakeman et al., 2006). In these steps, the available information is typically used for: identifying a model structure, and estimating its parameters. The identified model structure defines which quantities in the model (state variables and parameters) are known, which are unknown and need to be estimated, and how they are related. For short, we refer here to different model structures as different models. We refer to a specific model as a vector function $\boldsymbol{f}$ that receives the vector of parameter values $\boldsymbol{\theta}$, which is typically time-invariant and estimated using data, and inputs $\boldsymbol{u}$ that are system drivers and may be varying in space $(\boldsymbol{x})$ and time $(\boldsymbol{t})$. In explicit form

$\boldsymbol{y}(\boldsymbol{x}, \boldsymbol{t})=\boldsymbol{f}(\boldsymbol{u}(\boldsymbol{x}, \boldsymbol{t}), \theta)$

where $\boldsymbol{y}$ represents model outputs that, like $\boldsymbol{u}$, may vary in space and time. Bold notation indicates variables may be vectors rather than just scalars. In general, since a model is simply a tool to express what we think we know, $\boldsymbol{f}, \boldsymbol{\theta}, \boldsymbol{u}$, and therefore $\boldsymbol{y}$ are all likely to be uncertain. Every aspect of any particular model will differ from observations to some extent - they are all prone to error.

This is a very general definition of models that covers data-based, theory/process-based, and conceptual modeling approaches but for an indicative list of model families and features covered by (1), see Jakeman et al. (2006, p. 606). We note that data-based models are often used in situations where data volumes are copious compared to problems requiring theory-based or conceptual models. Nevertheless, the issue in data-based modeling is also to use a model structure $f$ and parameters $\theta$ to establish a relationship between $\boldsymbol{u}$ and $\boldsymbol{y}$ that can reproduce aspects of interest of the observed output behaviour of the model. Likewise with so-called integrated models consisting of linked model components (see Kelly (Letcher) et al., 2013 for five commonly used types in environmental applications), equation (1) remains relevant despite the fact that the form of $\boldsymbol{f}$ may be very complicated, and parameters may be defined for each sub-model separately rather than all at once.
Parameter values $\theta$ may be measured, where they correspond to observable properties of a system, or estimated (also known as calibration) from measured outputs by invoking an objective function that optimises constrained errors between the model and measured outputs, in what is referred to as an "inverse problem" - as opposed to the forward problem of simulating the model outputs given the parameters. Given errors in both observations and model structure, model and measured outputs do not perfectly match, resulting in uncertainty in the estimated parameters, and "residual" errors between the estimated and observed outputs. There are typically trade-offs between minimizing different aspects of the residual errors, such that the parameter estimation task itself is commonly seen as a multi-criteria problem (Efstratiadis and Koutsoyiannis, 2010; Gupta et al., 1998). Depending on the purpose of the analysis, it is recognized as good practice to work with multiple model structure hypotheses (Clark et al., 2011; Jakeman et al., 2006), and to quantify the total uncertainty in outputs resulting from using multiple models, uncertain parameters, estimation procedures/criteria, and residual errors. Ultimately, equifinality is unavoidable (Beven, 2006): given that errors cannot be eliminated or fully characterised, it is always possible to conceive of multiple different model structures and different parameter vectors that provide an $a c$ ceptable fit to observed data.

Nevertheless, in the context of making best use of available information, a modeler seeks to reduce uncertainty as much as is reasonably possible or required. There are four key, complementary methods for measuring how well uncertainty has been reduced (Fig. 1). Firstly, quantifying the uncertainty in outputs (or some function of them) provides a direct indicator, but is dependent on how well uncertainty in model structure, parameters, and residuals has been quantified (Refsgaard et al., 2007). Secondly, comparing observed and modeled outputs can be used in several ways. Amongst others, predictive accuracy provides a measure of model performance (see Bennett et al., 2013 for metrics and methods). The information supplied by a model can be quantified (Nearing and Gupta, 2015). If error analysis shows systematic rather than randomly distributed residuals, this typically indicates a problem with the model structure which, in general, leads to bias in the parameter estimates. Evaluating model adequacy is a substantial task of its own (Gupta et al., 2012). Given one or more model structures, a modeler is generally, in fact primarily, interested in how data has helped reduce uncertainty in parameters specifically. Thirdly therefore, the modeler can quantify the uncertainty in parameters, including the covariance describing how the parameter estimates relate to one other (Checchi et al., 2007; Thyer et al., 2009; Vrugt, 2016). With a suitable model structure, more data should typically result in smaller parameter uncertainty. However, this will not occur if the right type of data is not collected, which is the problem best tackled by investigating parameter identifiability.

It is important to understand the specific contribution of parameter identifiability analysis even, or especially, if one does not understand how to perform it. Parameter identifiability analysis focuses on whether it is possible to identify a unique vector of parameter values for a given 
model structure, or whether multiple parameter values will fit the data equally well. Non-identifiability means the modeler does not have the information needed to choose between alternative models (Rothenberg, 1971). Large parameter uncertainty is often indicative of non-identifiability, and reducing parameter uncertainty indicates that the situation has improved. The idea of "poor" identifiability is frequently approached from a parameter uncertainty perspective. Strictly speaking, however, identifiability analysis is specifically interested in the yes/no question of whether unique parameter values could be identified. It can be used both before and after data collection. Identifiability analysis before data collection can check whether the right type of data will be collected. Identifiability analysis after data collection can check whether the right type of data is being used in the parameter estimation process. It is therefore worth noting that even when the modeler's aim is to quantify parameter uncertainty rather than to estimate a single parameter vector, it is still useful to test whether a unique parameter vector could ideally be identified, in order to check whether mismatch between the model structure and type of data used is contributing to parameter uncertainty.

The power of identifiability analysis comes from focussing on a tightly defined mathematical problem - analyzing the relationship between knowns and unknowns in a given model structure. There is a simple rule when solving a linear system of equations that the number of unknowns should be less than the number of observations - otherwise the problem is "underdetermined" and "ill-posed", and an infinite number of solutions is possible. This simple rule cannot be easily applied in more complex models, and identifiability analysis provides some alternatives. It is common sense that a modeler should know whether their linear problem is underdetermined and what they should do about it. Similarly, it should be common sense for modelers to know whether their model structure is non-identifiable, and whether it matters for the purpose of their analysis.

This article aims at providing an introductory overview to key ideas of identifiability analysis. The field of identifiability has been extensively researched in various disciplines, including numerical examples in psychology in 1919 (Thomson, 1919) and independent early development of theory in econometrics and system identification in the 1950s and 1970s (Bellman and Åström, 1970; Koopmans and Reiersol, 1950). There is a number of other key reviews (Beck, 1987; Dobre et al., 2012; Godfrey and DiStefano III, 1987; Miao et al., 2011; Walter and Pronzato, 1996). This article differs from existing reviews by seeking to provide an accessible introduction to encourage the environmental modeling community to think more systematically and strategically about what type of information is needed to estimate parameters in their model, and increase adoption of the tools of identifiability analysis. The ultimate aim is therefore to improve research and management outcomes by fostering reflection on whether the selected model structure and available data are indeed appropriate to the problem at hand.

In Section 2, this paper first discusses the sources of non-identifiability within the scope of parameter identifiability analysis and then introduces fundamental concepts and methods underlying identifiability analyses. Building on these concepts, Section 3 discusses how these methods can be used in practice. Section 4 contains the conclusions.

\section{Fundamental concepts and methods}

\subsection{Sources of non-identifiability}

This paper seeks to capture key distinctions within the existing identifiability literature. We distinguish between three high-level sources of parameter non-uniqueness.

Source I is the model structure, including conceptualisation of the system, equations used to represent it, and optimisation objective function or model of the error structure (where applicable, see Sections
2.2 and 2.3). Parameter non-uniqueness can occur simply because of the choice of which quantities in the model are selected for observation. This is referred to as structural non-identifiability. The use of non-identifiable equations can be determined before having any input, internal state or output data. After data are available, identifying structural nonidentifiability can show that eliminating non-uniqueness requires data about different quantities in the model, or adoption of a different model structure that is identifiable with the type of data available.

Source II is the input, forcing dataset. Activation of different dynamics within a model depends on the inputs to the model, including initial and boundary conditions. If dynamics related to a parameter are not activated, then no information will be available to estimate that parameter. This is also referred to as persistence of excitation of the model dynamics. Given a data collection plan, it is possible to ascertain whether the forcing data suffices to make a parameter identifiable and it may also be possible to identify what data needs to be collected in order to successfully estimate parameters. After data are available, identifying lack of activation indicates that eliminating non-uniqueness requires observations in different experimental conditions or a different environmental context. This is particularly crucial in climate change applications, where the model is asked to make predictions in environmental conditions that are not reflected in historical data (Milly et al., 2008).

Source III consists of model and observation errors. Random noise or structural errors give rise to parameter uncertainty, which may or may not be quantifiable. More than one alternative model or parameter vector may be plausible, so by definition a unique parameter vector cannot be identified. It can, however, be useful to identify the most plausible parameter vector but this may not be possible due to the characteristics of the errors - the interaction of errors with model structure and forcing dataset and errors mean that several parameter vectors provide identical performance, according to the selected performance metrics. On the other hand, it is more common to have problems due to model structure, forcing dataset or large parameter uncertainty around the most plausible parameters, but it can still be useful to investigate how measurement errors can affect parameter estimation before embarking on expensive data collection processes - or afterwards, to help diagnose the source of problems and identify opportunities for improvement.

Consider an obvious example of non-identifiability involving estimation of parameters related to snow processes in a hydrological model. If no information is collected about snowfall, accumulation and melt, then unless other measurements can provide some indirect information it is likely that the relevant parameters are non-identifiable (Source I). If there is no snowfall within the period measured, then snowfall-inducing dynamics will not have been activated, so parameters cannot be estimated in that case (Source II). If snowfall is measured, but with large uncertainty, then the parameters will be uncertain. Depending on the properties of the errors, it may be impossible to identify unique parameters that best fit the data (Source III).

Source I is traditionally considered the core concept of so-called theoretical, "structural or a priori identifiability" (Bellman and Åström, 1970; Dobre et al., 2012). The remaining sources fall in the domain of so-called "practical identifiability". Structural identifiability involves analysis of the equations of the model and can be undertaken without observational data. Practical identifiability is based upon analysis of the ability to estimate parameters from observational data. Structural nonidentifiability implies practical non-identifiability. If the equations are not identifiable, then it does not matter under what conditions the data are collected, how much is collected, or how accurate they are - the model structure determines it will not be possible to uniquely estimate parameters in practice. Structural identifiability, however, does not imply practical identifiability. If the model structure theoretically allows parameters to be estimated, one still needs to have the appropriate data to achieve this. 


\subsection{Identifiability of model equations}

Focussing on model equations, a model structure can be said to be globally identifiable at $\boldsymbol{\theta}=\boldsymbol{\theta}^{*}$ if, for a given input $\boldsymbol{u}(\boldsymbol{x}, \boldsymbol{t})$ and measurable system output $\mathbf{y}(\boldsymbol{x}, \boldsymbol{t})$, all other parameter value vectors will yield different output vectors (Ljung and Glad, 1994). Conversely, only a single vector of parameter values will perfectly match a given set of inputs and outputs. Formally, a model is globally identifiable if:

$\boldsymbol{f}(\boldsymbol{u}(\boldsymbol{x}, \boldsymbol{t}), \theta)=\boldsymbol{f}\left(\boldsymbol{u}(\boldsymbol{x}, \boldsymbol{t}), \theta^{*}\right) \Leftrightarrow \theta=\theta^{*}$

Otherwise the model is said to be non-identifiable.

Additionally, a model structure is said to be locally identifiable if there is a neighbourhood of values around $\theta=\theta^{*}$ where this condition holds (Ljung and Glad, 1994). In that case, other solutions can only occur in separated neighbourhoods, such that there is usually a finite number of solutions. For example, consider quadratic functions; for the equation $y=x^{2}$, the value for $x$ is locally but not globally identifiable; it has two values $x=+\sqrt{y}$ and $x=-\sqrt{y}$.

If a solution is locally as well as globally non-identifiable, there will instead be an infinite number of solutions. This can be explained easily for under-determined linear systems. Suppose we have the equation $a x_{1}+b x_{2}=y$ with unknowns $a$ and $b$, and one observation with values for $x_{1}, x_{2}$ and $y$. With one observation and two unknowns, the problem is under-determined and there is an infinite number of combinations of $a$ and $b$ that would fit the observation. The problem is both locally and globally non-identifiable.

Identifiability is by definition a binary problem, i.e., a parameter is either identifiable or non-identifiable given the model structure and the type of data available. Although the equations above are strict mathematical definitions, the underlying idea here is to understand whether identifying unique parameters is theoretically possible. Whilst in practice, numerical errors in computing might need to be accounted for, the idea of identifiability proper is not concerned with whether parameter values are approximately equal. As long as there is some difference, it is theoretically possible to differentiate between alternative parameter vectors, for example by increasing the sample size of data collected, which then becomes a question of uncertainty reduction. If the parameter vectors cannot be distinguished even in theory, this is important information for a modeler.

Throughout Section 2, two simple models are used as examples. We represent them firstly as equations, because identifiability depends solely on the mathematical relationship between variables, not on their real-world interpretation. To help understand the implications of the mathematical relationship, we use two different interpretations. Firstly, they are single rate equations that describe the relationship between biomass concentration and oxygen consumption rate $\left(r \mathrm{~kg} \mathrm{O} / \mathrm{m}^{3} \mathrm{~s}\right)$ in two different conditions. In a second, more informal interpretation, we consider a restaurant where a waiter is trying to guess, for a table of two regular customers, how much each person usually gives as a tip ( $r$ in USD).

The equation for the first model describes one species $(X \mathrm{~kg}$ biomass $/ \mathrm{m}^{3}$ ) with parameters for biomass growth $\left(\theta_{1}\right)$ and maintenance $\left(\theta_{2}\right)$, or a case where the table for two pays a single bill on a company card ( $X$ USD) but pool their cash tips, resulting in different tipping percentages $\left(\theta_{1}\right.$ and $\left.\theta_{2}\right)$. Thus

$r=\left(\theta_{1}+\theta_{2}\right) X$

It is clear that an infinite number of combinations of $\theta_{1}$ and $\theta_{2}$, with the same sum, will produce the same value of $r$. Hence, no unique pair of parameter values can be found, and we say that the parameters $\theta_{1}$ and $\theta_{2}$ are non-identifiable. This is an example of a priori or structural non-identifiability (Source I). Knowing the amount of biomass and oxygen consumption does not allow us to uniquely identify the values for biomass growth and maintenance. Knowing the size of the bill and the tip does not allow the waiter to determine how much each person contributes.
The equation for the second model describes a rate equation for two species $X_{1}$ and $X_{2}$ with biomass parameters $\theta_{1}$ and $\theta_{2}$, or a case where the table for two paid separate bills:

$r=\theta_{1} X_{1}+\theta_{2} X_{2}$

Then the model is structurally identifiable, in that both $\theta_{1}$ and $\theta_{2}$ can be uniquely estimated, with two measurements, as long as $X_{1}$ and $X_{2}$ are not zero.

If $X_{1}$ or $X_{2}$ is zero, we have no information about the respective parameter. With only one measurement, we would have no information about the dynamics between the variables - how they change relative to one other (it would be an underdetermined linear system). This is an example of non-identifiability due to insufficient excitation of model dynamics (Source II).

If $r$ and/or $X_{1}$ and $X_{2}$ is measured with errors, or the equation is not accurate, then with two measurements, the parameters will still fit perfectly, but with biased parameters ("overfitting" to the noise). With three measurements or more, the observations will not perfectly fit the model (it would be an overdetermined linear system). Best fit solutions need to be found instead, so $\theta_{1}$ and $\theta_{2}$ will be uncertain, and there may not be a well-defined best fit solution either. This is an example of nonidentifiability due to observation errors (Source III).

Knowing oxygen consumption and both biomasses allows us to estimate the oxygen consumption rates, but only if there is a biomass to measure, and all the values are accurately measured. Knowing each person's bill and the total tip is sufficient for the waiter to estimate the tipping rate for each person, as long as they actually had a bill, and the waiter remembered the totals accurately.

Analysis of identifiability of model equations can be carried out in two ways, i.e. testing whether identical outputs imply identical parameters, or testing that different parameters yield different outputs, which is referred to as output distinguishability (DiStefano III and Cobelli, 1980). In practice, such analytic approaches commonly involve transforming the model in order to facilitate analytical manipulation. The easiest approach to use depends on model properties, and in some cases computer algebra can be useful (e.g. Chiș et al., 2011; Karlsson et al., 2012; Saccomani and Bellu, 2008). Except for relatively simple models, this analytical problem is usually hard to solve in this general form, but can be extremely useful. If an analytical approach shows a model is non-identifiable, it will generally reveal why, and hence suggest possible remedies (Stigter et al., 2017).

This introductory overview will not provide a detailed discussion of analytic methods for identifiability, as they typically require an advanced knowledge of mathematics. In future, improvement in user friendliness of software may enable wider use of these methods. In the meantime, we refer the reader to the following useful literature. Walter and Pronzato (1996) provide a summary of four key approaches involving: systems of derivatives from a power series/Taylor series expansion, coefficients of series using Lie derivatives, identification of local state isomorphisms, and differential algebra. Equivalent approaches for linear systems involve Markov parameters, coefficients of Laplace transforms and identification of similarity transforms. Norton (1980) and Norton et al. (1980) discuss transformation and analysis in terms of eigenvectors/normal modes, and linear regressive re-parametrization is presented in Keesman and Doeswijk (2009) and Keesman (2011). Stigter et al. $(2017,2015)$ show that combinations of analytical and numerical approaches may improve computational efficiency. A review by Miao et al. (2011) also includes approaches that directly use our definition of model equations identifiability, formulate it as a constraint satisfaction problem and use the implicit function theorem. Other useful overviews include Cobelli and DiStefano (1980), Norton (1982), and Godfrey and DiStefano III (1987). 


\subsection{Identifiability with an objective function}

\subsubsection{Parameter estimation}

Ideally, estimating parameters would involve a straightforward solution of an inverse problem. The values of parameters could be mathematically deduced from observations. This is, for example, the case for a linear system of equations, as already illustrated for equation (5). However, outside this narrow case, model inversion tends to be approached as an optimisation problem, for example when the model consists of non-linear, complex equations, or errors are present in data.

Parameter estimation commonly involves optimizing an objective/ loss function $m(\theta)$ (see overview in Bennett et al., 2013; Marsili-Libelli, 2016), sometimes with constraints on the solution. It quantifies how well the model and parameters fit the data. The optimal parameter values $\theta^{*}$ therefore minimize $m(\theta)$.

A common, relatively simple objective function is the mean squared error (MSE). Let $\boldsymbol{f}$ be a model predicting $n$ outputs at locations $\boldsymbol{x}$ and times $\boldsymbol{t}$, i.e. $\boldsymbol{y}(\boldsymbol{x}, \boldsymbol{t})=\left(y_{1}, \ldots, y_{n}\right)^{T}=\boldsymbol{f}(\boldsymbol{u}(\boldsymbol{x}, \boldsymbol{t}), \theta)$. Given a set of $n$ data $\boldsymbol{d}=\left(d_{1}, \ldots, d_{n}\right)^{T}$, for example, noisy data $\boldsymbol{d}=\boldsymbol{f}\left(\boldsymbol{u}(\boldsymbol{x}, \boldsymbol{t}), \theta^{t}\right)+\varepsilon$, with independent measurement error $\varepsilon=\left(\varepsilon_{1}, \ldots, \varepsilon_{n}\right)^{T}$ obtained using some unknown true parameter vector $\theta^{t}$, the MSE $m$ for a parameter vector $\theta$ is

$m(\theta)=\frac{\sum(\boldsymbol{y}(\theta)-\boldsymbol{d})^{2}}{n}$

In many statistical methods, the objective function used is the likelihood that a parameter vector is the true value given the observations. In that case, the optimisation involves maximizing the likelihood, and therefore produces the "maximum likelihood estimate" of the parameter values (MLE). The likelihood needs to capture the distribution of errors, notably the covariance of the measurement errors, including autocorrelation and heteroscedasticity (e.g. Schoups and Vrugt, 2010).

Bayesian parameter estimation uses a prior probability distribution in addition to the likelihood, and optimisation yields "maximum a posteriori probability" (MAP) estimates of the parameter values (Stuart, 2010). However, Bayesian statistics typically focuses on the use of distributions rather than "point" estimates, such that it is more common to report the mean or median parameter value and its uncertainty, rather than the MAP estimate. This is further discussed in Section 2.3.6.

\subsubsection{Identifiability as a unique optimal solution}

Optimizing an objective function in effect provides some flexibility in parameter estimation. Instead of the model output having to fit the observations exactly, some deviation is permitted. This poses a problem in terms of Equation (2) - more than one set of parameter values will fit given inputs and outputs if we allow some deviation. Optimisation tries to solve this by quantifying the deviation with an objective function and minimizing that deviation rather than specifying how much is permitted. In circumstances where a perfect fit is not possible, we settle for the best available, and would ideally still like the solution to be unique. For parameters to be identifiable, however, the objective function should have a single optimal solution (Bellman and Åström, 1970). That is, instead of Equation (2), if our best available solution has an objective function value of $m(\theta)=m^{*}$, we want to know that:

$m(\theta)=m\left(\theta^{*}\right) \Leftrightarrow \theta=\theta^{*}$

subject to the constraint that $m(\theta)=m^{*}$

In an optimisation context, uniqueness and identifiability therefore depend on the objective function used. Some objective functions can be essentially handicapped to lead to unique parameter vectors, if used individually. For example, if our objective function was simply the sum of residuals (also referred to as the bias), then simplification of equation (7a) below shows that data has a limited effect on identifiability - $\boldsymbol{d}$ only appears in the constraint. $\sum(\boldsymbol{y}(\theta)-\boldsymbol{d})=\sum\left(\boldsymbol{y}\left(\theta^{*}\right)-\boldsymbol{d}\right) \Leftrightarrow \theta=\theta^{*}$

subject to the constraint that $\sum \boldsymbol{y}(\theta-\boldsymbol{d})=m^{*}$

$\sum \boldsymbol{y}(\theta)=\sum \boldsymbol{y}\left(\theta^{*}\right) \Leftrightarrow \theta=\theta^{*}$

subject to the constraint that $\sum \boldsymbol{y}(\theta)=m^{*}+\sum \boldsymbol{d}$

Specifically, if we consider a hydrological model, where the model output consists of river flows, equation (7b) says that parameters are only identifiable if the total river flow with least bias can only be obtained by a single parameter vector. This is unlikely to occur for many models - there are typically many combinations of parameter vectors that could achieve a given total flow. The objective function in equation (6) is less likely to have that problem (equation (8)), though identifiability still depends on the model structure and the precise observations used.

$\sum(\boldsymbol{y}(\theta)-\boldsymbol{d})^{2}=\sum\left(\boldsymbol{y}\left(\theta^{*}\right)-\boldsymbol{d}\right)^{2} \Leftrightarrow \theta=\theta^{*}$

subject to the constraint that $\sum(\boldsymbol{y}(\theta)-\boldsymbol{d})^{2}=m^{*}$

Equation (6) can also be extended to a case where multiple objectives are of interest. In a multi-objective parameter identification setting, the aim is to check the uniqueness of a given vector of parameter values on the "Pareto front" - a compromise solution between objectives where no other parameter values provide better performance on every objective of interest at once (e.g. Maier et al., 2019). In this case, Equation (6) can simply be written in vector form. Equation (9) expands the vector notation for a case with only two objectives. For a parameter vector on the Pareto front with $m_{1}(\theta)=m_{1}^{*}$ and $m_{2}(\theta)=m_{2}^{*}$, the problem is identifiable if:

$m_{1}(\theta)=m_{1}\left(\theta^{*}\right) \Leftrightarrow \theta=\theta^{*}$

$m_{2}(\theta)=m_{2}\left(\theta^{*}\right) \Leftrightarrow \theta=\theta^{*}$

subject to the constraint that $m_{1}(\theta)=m_{1}{ }^{*}$ and. $m_{2}(\theta)=m_{2}{ }^{*}$

Solving any of equations 7-10 is usually difficult, even if we know the value of $m^{*}$ ahead of time. Instead, it is easier to think of the problem as simply exploring whether there is a single global optimum. "Global" optimality refers to being the best solution across the entire parameter space, as opposed to "local" optimality, meaning that the solution is only better than the parameter values in its immediate neighbourhood in the parameter space. Two fundamental approaches for examining global optimality, and therefore identifiability, involve visualizing the response surface and examining derivatives. These are discussed in the next sections.

While it may seem like evaluating identifiability with an objective function requires data, this is not necessarily the case. Identifiability can be assessed without observations by using "synthetic data" (e.g. Shin et al., 2015). Assumptions are made about the form of the input data, and the model is run in order to obtain "exact" error-free output data. In controlled experiments, the input corresponds to the intended experimental conditions. In other settings, the inputs correspond to the conditions in which data are expected to be obtained. With error-free input and output data, it should be possible to obtain a perfect value of the objective function, i.e. $m(\theta)=0$, and any non-uniqueness in parameters arises only from model equations (including objective function) and properties of the input (Sources I and II), not from noise or errors (Source III). Such an experiment typically assumes that the model structure is a perfect representation of the underlying system.

It should be noted that when evaluating identifiability with data, the analyst also needs to think about whether they can efficiently locate that solution. It is likely that the model is not identifiable if different solutions are reached with repeated runs of optimisation algorithms there is more than one global solution, for example with different seeds for stochastic algorithms or different initial values for deterministic algorithms (Shin et al., 2015). Optimisation can, however, also fail for other reasons. Local optima are a common problem. Iterative optimisation algorithms require the use of stopping criteria that affect the 

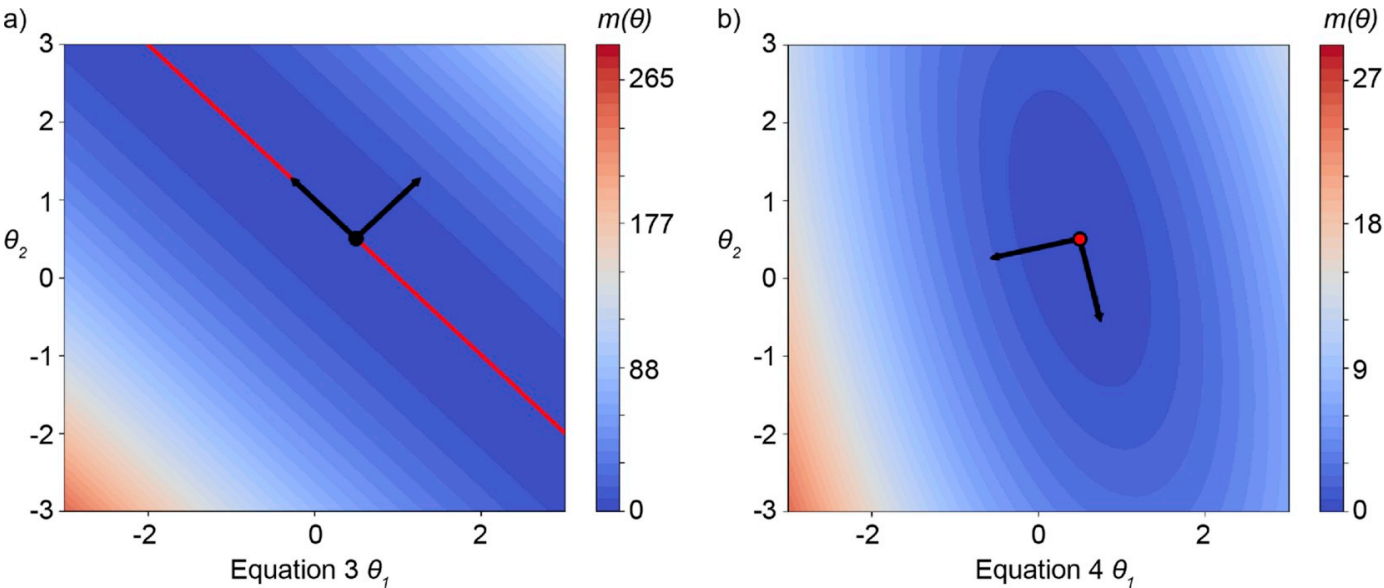

Fig. 2. Response surface of the consumption rate $r$ as a function of biomass growth and maintenance for the models given by Equation (3) (plot a) and Equation (4) (plot $b$ ). Red point/line represents parameters that minimize the MSE. The black dot represents the truth parameters that generated the data. The arrows represent the eigenvectors of the Hessian of the misfit m (see discussion in Section 2.3.4). (For interpretation of the references to colour in this figure legend, the reader is referred to the Web version of this article.)

accuracy of the final "optimal" solution, and in many environmental modeling problems identifying the global optimum is not guaranteed, even if it does exist.

\subsubsection{Visualizing the response surface or fitness landscape}

Rather than analyzing equations, visualization can be used to investigate identifiability. Taking a geometric point of view, the value of the dependent "response" variable is interpreted as a surface, varying dimensionally as a function of the independent variables, usually parameters. This is referred to as a "response surface" (Box and Draper, 1987), and can be used to visually evaluate whether different parameters give similar outputs (as required by equation (2)). When the response variable is an objective function, the response surface is referred to as an "error surface" or "fitness landscape" (also see Maier et al., 2019, 2014), as shown in Fig. 2. The optimisation algorithm can be thought of as navigating this landscape in order to find the optimal (highest or lowest) point.

Suppose we are at the top of a mountain. To be able to say that we are at the single highest point of a mountain range, we would need to check that we are actually on a peak - not on a plateau or ridge-line, and that no other peak in the range is the same height. That is, visually to assess whether multiple points are optimal, we need to check for two problems:

1. Flat surfaces, in some direction, such that points in that direction with different parameter values have the same output value. These cases are both locally and globally non-identifiable.

2. Distinct peaks with an equal objective function value. These cases are locally identifiable, but globally non-identifiable.

Note that a flat surface is not the same as having a flat slope at the top of the mountain (zero gradient). The slope might allow us to stand vertically at the top of a peak, but any step to the side brings us down the mountain rather than walking along a plateau or ridge. This is further discussed in Section 2.3.4.

A flat surface could also occur in any direction, for example northwest, not just along the main axes (north-south and east-west). The surface might be flatter in a direction that varies multiple parameters at once rather than when varying a single parameter at a time. The extent to which the combined effect of simultaneously varying parameters on an objective function differs from varying the parameters separately (one at a time) is indicative of "parameter interaction". It is an important feature to look out for because it tends to be easily overlooked, especially if relying on "one-at-a-time" analyses (Saltelli and Annoni,
2010).

Fig. 2 (a) plots the unweighted mismatch (equation (6)) between the model in equation (3) and two observations of the consumption rate $r$ for $X=1$ and $X=2$, with the true value of the parameters vector as $\theta^{t}=(0.5,0.5)^{T}$ - the black dot. The red line represents the possible values of parameters that minimize $r$. Because of the lack of identifiability that we observed in the previous section, an infinite number of parameter combinations can exactly reproduce the data.

Fig. 2 (b) plots the unweighted mismatch between the model in equation (4) and two observations of the consumption rate $r$ for $X_{1}=0.5, X_{2}=0.5$ and $X_{1}=-1, X_{2}=0$ at $\theta^{t}=(0.5,0.5)^{T}$. The surface shows a single optimal low point, corresponding to the optimal minimum (other objectives could yield a single peak, corresponding to an optimal maximum). Around the peak are concentric ellipses of parameter values with the same objective function or likelihood. A model is identifiable if there are no flat surfaces at an optimum and, in the case that distinct optima exist, there is only one global optimum.

The response surface can easily be visualized for models with one or two parameters. When models have larger numbers of parameters, visualizing selected one- and two-dimensional subspaces of the higherdimensional parameter space can be useful. The response surface in such subspaces illustrate the change in the objective function as a function of one and two parameters respectively, keeping others constant. For instance, a one-dimensional sub-space corresponds to a crosssection through a landscape. Razavi and Gupta (2015) provide examples of the response surface of a complex, high-dimensional model when visually viewed within different two-parameter sub-spaces (see Fig. 3 therein), by which the modeler can learn about a range of features in the response surface from small-scale features such as roughness and noise to large-scale features such as trends and multi-modality.

One-dimensional projections (also known as "dotty plots", Beven (2006), or the "profile likelihood", Raue et al. (2009)) and two-dimensional projections (e.g. Shin et al., 2015) are also useful for models with large numbers of parameters. Dotty plots show the values of one or two parameters while varying all other parameters. This can help suggest potential flatness and interactions between pairs of parameters, globally across all parameter space, rather than at any particular point (see e.g. Shin et al., 2015). For our two example models, Fig. 3a and c shows that a large range of parameters have approximately the same objective function value, whereas in Fig. $3 \mathrm{~b}$ and $\mathrm{d}$ there is a well-defined optimum. Visual inspection helps to build understanding of the response surface of a model. In practice, it is often necessary, however, to use numerical or analytical tests of the objective function, for example when a model has many parameters (e.g. $>20$ ), or if optima are 
a)

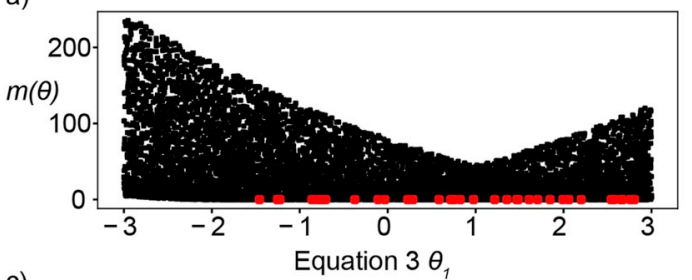

c)

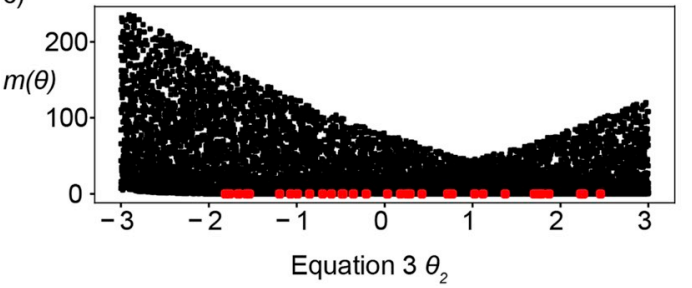

b)

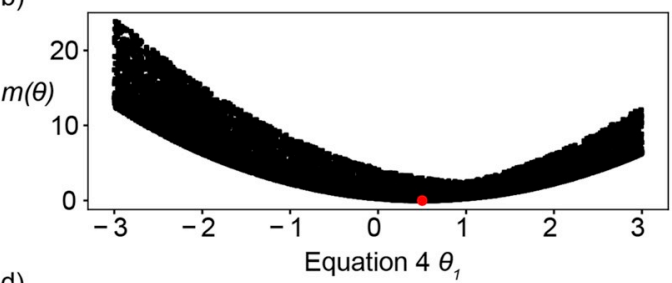

d)

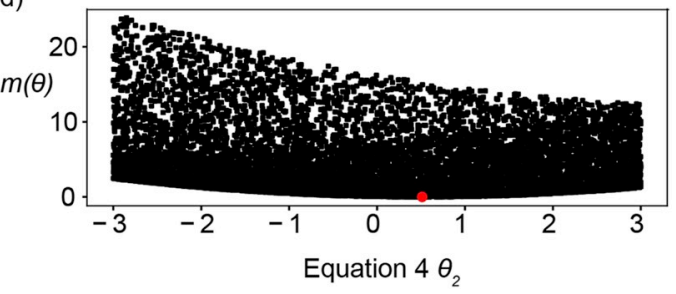

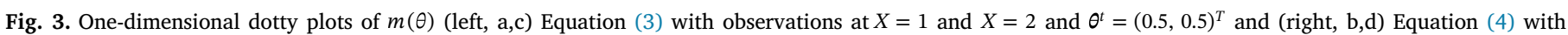

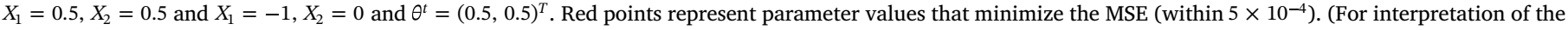
references to colour in this figure legend, the reader is referred to the Web version of this article.)

not visible in the plot because they lie in a narrow hole (or on top of a narrow peak). It is typical to use multiple methods for checking identifiability (Balsa-Canto and Banga, 2011).

\subsubsection{Examining the value of derivatives}

Absence of flat surfaces and distinct optima can be more directly assessed by examining the gradient of the response surface at points across parameter space. Intuitively, one might think a flat surface would be identified by a zero gradient, but the situation is more complex when evaluating the gradient at a point.

For a model with only one parameter, a minimum can be identified as a parameter value where the first derivative (gradient) is zero (i.e. a stationary point) and the second derivative is positive, such that the objective function increases away from the minimum (see Fig. 4a-c). The gradient is the slope at a point, and the second derivative is its curvature. For a maximum, the second derivative should be negative, such that the objective function decreases away from the optimum.

If, at a point, the first derivative is zero (i.e. a stationary point), and the second derivative is zero as well, but the third derivative is non-zero (i.e., an inflection point), the point is a "saddle point" not an optimum (see Fig. 4d-f). If there are multiple parameter values with gradient equal to zero, there are multiple local optima (see Fig. 4g-i).

Non-identifiability where the objective function is flat therefore occurs when the first, second and third derivatives are all zero, as well as further "higher order" derivatives, though in practice they may have little effect. The flatness is referred to as lack of sensitivity, and the derivatives allow us to check for insensitive parameters at a particular point. If examining the derivatives shows that there are multiple local optima, then their objective function values need to be compared to see whether there is more than one global optimum. Examining the derivatives can therefore help identify both the problems visualized with the response surface.

For a model with more than one parameter, partial derivatives are calculated with respect to each parameter separately. This results in a gradient vector, with the partial derivative for each parameter. For an optimum, the first partial derivatives should be zero, and a matrix of second partial derivatives is then constructed, considering pairs of parameters together (including second partial derivatives of each parameter with respect to itself). This is known as the Hessian matrix. The Hessian describes how the slope of the response surface changes in every direction, in other words its curvature. To illustrate this, Fig. 5 shows a hypothetical response surface that features a global maximum and a local maximum, a saddle point in between, and a flat area. These four points are all stationary, meaning the gradient vector (first-order partial derivatives) is zero. However, the Hessian matrices at these four points have significantly different properties. If all the second partial derivatives are non-zero, the response surface is not flat. If one is zero, then the response surface may be flat, if the higher order derivatives are also zero. In particular, if any third-order derivative is non-zero, then there is a saddle point rather than a flat surface.

As shown in the figure, the Hessian at a stationary point can be cast as a quadratic function fitted to the response surface at that point (a parabola, for a single parameter function). Mathematically, this is equivalent to using the first three terms of a Taylor series expansion. The quadratic illustrates how the Hessian contains information about the slope in all directions, not just along each parameter axis. If the Hessian is a diagonal matrix, the associated parabola is orthogonal (i.e. no pairwise interaction term in the quadratic function), and therefore there is no interaction effect between the parameters locally at that point. However, if the Hessian is non-diagonal, the associated quadratic is non-orthogonal as a result of interaction between the parameters. In Fig. $5 \mathrm{~b}$, the quadratics describing the local and global maxima both have significant parameter interactions. If the axes were compass directions, the surface around the local maximum would be flattest along a northwest-southeast axis, and steepest along a northeast-southwest axis. The global maximum is flattest along a northeast-southwest axis and steepest along a northwest-southeast axis.

The interaction effects at a stationary point can be summarised with an eigendecomposition, calculating the eigenvectors and eigenvalues of the Hessian, which respectively describe a set of orthogonal directions (an orthogonal basis), and the second partial derivative in that direction. The advantage of this transformation is that the first eigenvalue is the largest second partial derivative in any direction, and the last eigenvalue is the smallest. The response surface at a point may therefore be flat if the last eigenvalue(s) are zero, meaning that the second partial derivative is zero in the corresponding direction, and the Hessian is then referred to as singular. Conversely, the point is an identifiable minimum if all eigenvalues are positive, such that second partial derivatives are positive and the gradient and objective function increase in all directions. The Hessian is then referred to as non-singular and positive-definite. Similarly, the point is a maximum if all eigenvalues are negative, and the Hessian is referred to as negative-definite. If the eigenvalues have different signs, the point is a saddle point, which in a two-parameter case resembles a horseback riding saddle (see Fig. 5b). For more detail on the role and the numerical aspects of the Hessian computation, see for example Marsili-Libelli (1992), Seber and Wild (1989) and Marsili-Libelli et al. (2003).

The eigenvectors of the models in Equations (3) and (4) were also shown in Fig. 2. For Equation (3) the eigenvalues are 10 and 0 . The Hessian is semi-positive definite indicating that an optimum exists but it 
(a)

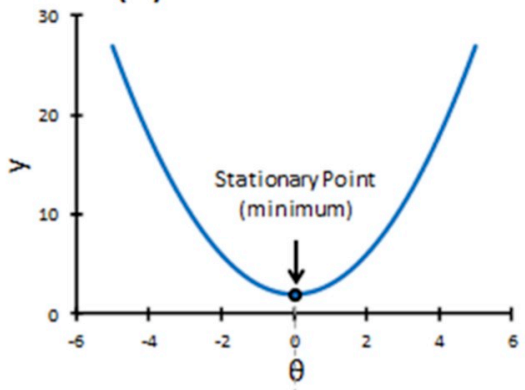

(b)

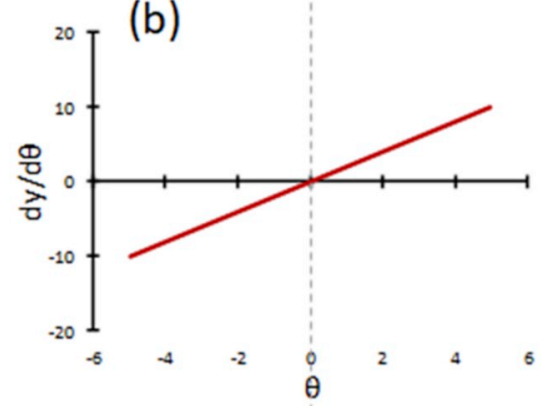

(c)

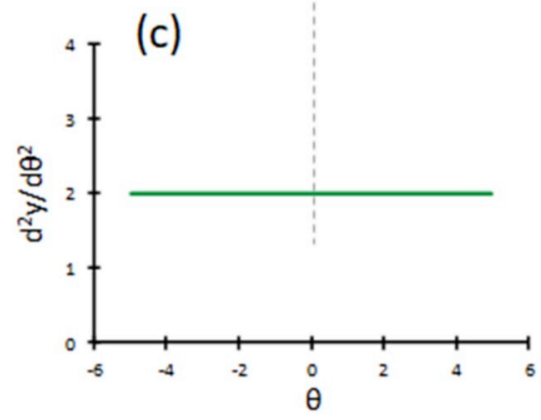

(d)
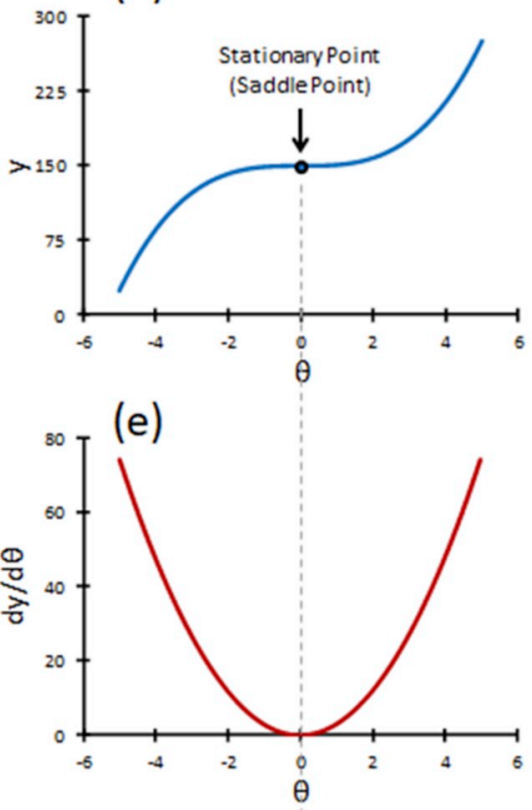

(f)

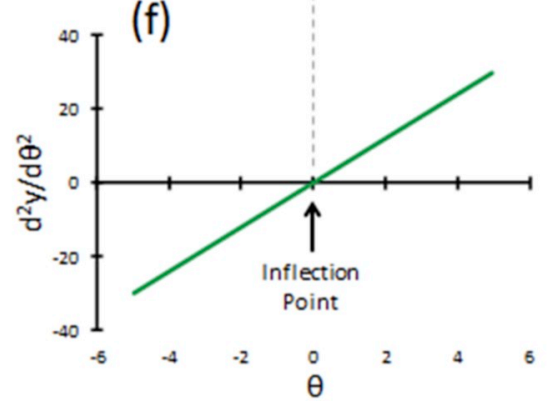

(g)
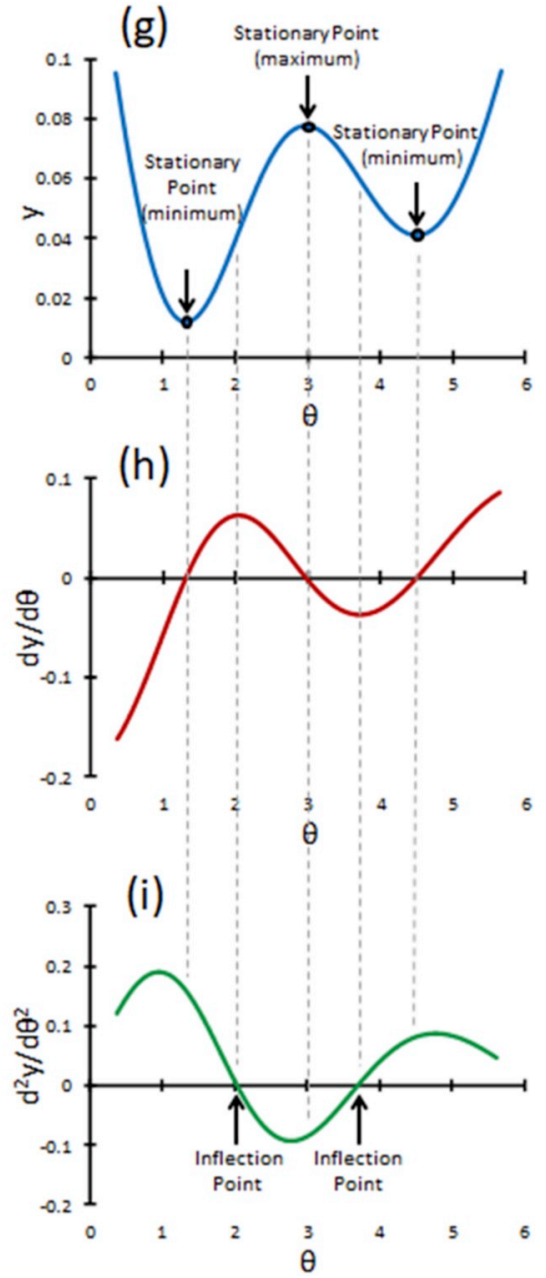

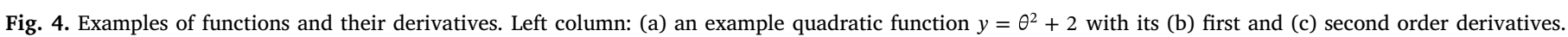

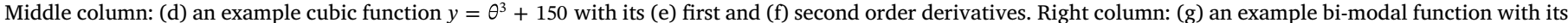
(h) first and (i) second order derivatives.

(a)

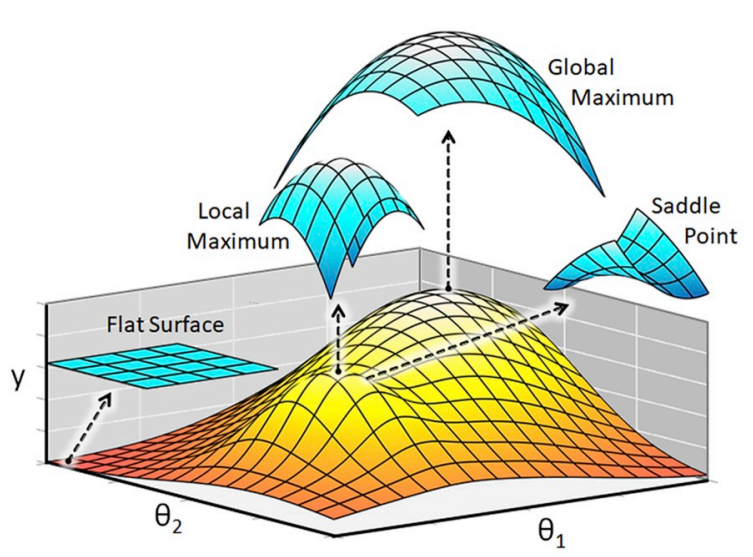

(b)



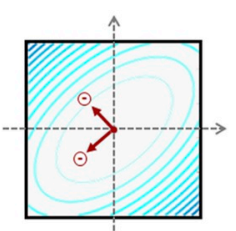

Global

Maximum

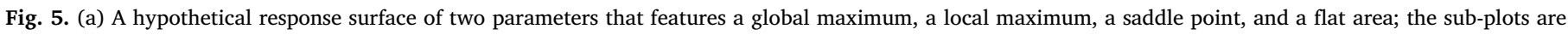

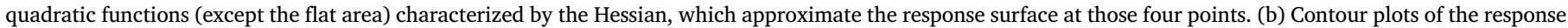

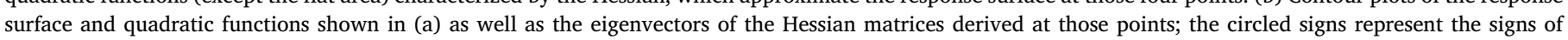
eigenvalues, such that a positive/negative value indicates that the surface in the direction of the corresponding eigenvector curves up/down. 
is not unique. For Equation (4) the eigenvalues are approximately 1.31 and 0.19 . The larger eigenvalue corresponds to the direction (eigenvector) along which the misfit changes the fastest.

In practice, the eigenvalues of the Hessian may not be examined directly. The determinant of the Hessian can be calculated as the product of eigenvalues (amongst other methods). If any of the eigenvalues are zero, the determinant will therefore also be zero, which also tells the analyst that the Hessian is singular and may have a flat response surface in some direction. In statistics textbooks, the reader may come across this approach in likelihood-based methods that analyse the observed Fisher Information Matrix (FIM) or the expected FIM. The observed FIM corresponds to the negative of the Hessian matrix when using the loglikelihood as objective function. The expected FIM averages over the likelihood function rather than using observed values. If the FIM is nonsingular, then the model structure is locally identifiable (Bellman and Åström, 1970; Rothenberg, 1971). In general, it is difficult to demonstrate singularity due to numerical errors. It is often better to perform a rank test with a singular value decomposition (SVD) of the sensitivity matrix, which consists of the partial derivatives of the model outputs with respect to each parameter (see e.g. Miao et al., 2011).

As the Hessian indicated, the objective function may also be flat due to interactions between parameters. The combined effect of varying two parameters may cancel out such that the objective function does not change. A variety of other indices have been proposed that measure the strength of interaction of parameters or its effect on identifiability (see e.g. Brun et al., 2001; Doherty and Hunt, 2009; Hill and Tiedeman, 2007; Sorooshian and Gupta, 1985). As an example, Active Subspaces provide a global indicator (Constantine et al., 2014), similar to how the eigendecomposition summarises the Hessian - if all singular values are non-zero and of similar magnitude then the system is likely to be globally identifiable. Alternatively, variance-based sensitivity analysis methods can quantify the combined effect of specific combinations of parameters, averaged across parameter space. However, sensitivity of a combination of parameters (i.e. strong interaction) does not necessarily mean that they are non-identifiable (Dobre et al., 2012). At any specific point, the interaction may not result in a flat objective function.

Derivative-based methods, including the techniques using sensitivity analysis, are relevant when the derivative can be calculated (whether analytically or numerically) and non-identifiability is more likely to occur due to flat surfaces than distinct optima; there are few optima to find and examine. Derivative-based methods may not be appropriate if there are numerical problems in the model or if the model output or objective function is characterised by discontinuities or many distinct solutions. In these situations, other approaches need to be used (including examining the response surface).

\subsubsection{Sensitivity versus identifiability}

Identifiability analysis (IA) is closely related to sensitivity analysis (SA). SA seeks to measure the sensitivity of a model response to perturbations in different model parameters. See Norton (2015) for an introductory overview of SA, Saltelli et al. (2000) as a comprehensive reference, and Razavi and Gupta (2015) and Pianosi et al. (2016) for recent reviews of the state of the art.

If the objective function is insensitive to a parameter, it means that the objective function is flat, and the parameter is not identifiable. However, one needs to distinguish between sensitivity and identifiability. Even if the objective function is sensitive to all parameters, it is not guaranteed that the parameters are identifiable for the reasons below.

SA aims to establish which parameters exert stronger (or weaker) controls on the model response, which is an attribute of the forward problem. In contrast, IA aims to establish which parameters are identifiable, given observations on the target response, which is an attribute of the inverse problem. Measuring sensitivity on an objective function combines attributes of the forward and inverse problems, but plays a "filtering role" that may obscure the information gained (Gupta and
Razavi, 2018).

"Local" sensitivity analysis (LSA) at a nominal point in the parameter space is based on partial derivatives at that point, similar to the approach discussed for identifiability in the previous section. The same condition therefore applies, that assessing identifiability using LSA of the objective function needs to be complemented by evaluation of other local optima in order to determine whether a single global optimum exists. LSA provides a limited view on sensitivity, only locally around the nominal point (Saltelli and Annoni, 2010). Optimal parameter values therefore need to be obtained before using LSA to assess identifiability with an objective function.

Alternatively, a wide range of methods have been developed that seek "global" sensitivity analysis (GSA), measuring sensitivity across the entire parameter space. Examples include derivative-based methods (Campolongo et al., 2007; Rakovec et al., 2014; Sobol' and Kucherenko, 2009), variance-based methods (Homma and Saltelli, 1996; Sobol', 2001), and variogram-based methods (Razavi et al., 2019; Razavi and Gupta, 2016a, 2016b), the latter bridging derivative- and variancebased methods by characterizing the response surface across the full spectrum of perturbation scales (Haghnegahdar and Razavi, 2017).

If a parameter has no effect on the objective function anywhere in parameter space, it also has no effect at the optimum, wherever it is located. However, even if a parameter is sensitive on average across the parameter space, it is not guaranteed that it is sensitive at the global optimum - the parameter still may not be identifiable.

GSA also allows evaluating the effect of parameters on model outputs, and therefore investigating structural identifiability. Similarly, if a parameter has no effect on the model output anywhere in parameter space, it is non-identifiable. As noted above, it is also common practice to use SA to identify interactions between parameters, while remembering that the absence (or presence) of interactions does not necessarily indicate (non-) identifiability. Sensitivity analysis is therefore an effective tool for identifying non-identifiability, but not for ruling it out. See Gupta and Razavi (2018) and Dobre et al. (2012) for further detail on commonalities and differences between SA and IA.

\subsubsection{Role of noise and systematic errors}

As presented in Section 2.3.2, the use of an objective function provides a mechanism for taking into account noise and errors in assessing identifiability - we can assess whether the best fit is only attainable using a single vector of parameter values. As equation (6) depends on the observed data, non-uniqueness may indicate that the properties of "errors" prevent uniqueness (Source III), in addition to which quantities are observed (Source I) and the conditions represented in the forcing data (Source II). By "errors", we mean any discrepancy between the modeled and observed systems. These errors can take many forms: noise or systematic errors in the measurement of either inputs or outputs, discrepancy between the model structure and modeled system, as well as misrepresentation of the properties of errors themselves, as captured by the objective function. We focus here on general principles rather than explaining the differences in effect of these different errors.

In addition to potentially affecting the identifiability of the optimal solution, errors influence the identifiability problem in another key way: the estimated parameters will generally change depending on the data used (and the objective function invoked). Samples of observations with different data errors result in different estimates of parameter values. Values of parameters will therefore remain uncertain even if a unique optimum solution can be identified when using noise-free data (Vanrolleghem and Keesman, 1996).

If the parameters are anyway non-unique, this provides a strong motivation for switching from optimisation-based to uncertainty-based parameter estimation. In that case, rather than aiming to identify a single parameter vector, the aim is to identify a set of many parameter vectors that all adequately fit the data, i.e. to quantify the uncertainty in parameters (see Matott et al. (2009) for a review of methods for 
quantifying uncertainty in parameters). As foreshadowed in Section 2.3.1, this is the approach preferred by Bayesian inference, which focuses on identifying a "credible region" of parameters - within which an unobserved parameter falls with a given (subjective) probability. It can also be approached more directly as a "set membership" problem, as notably used in the Generalised likelihood uncertainty estimation (GLUE) approach where only the set of "behavioural" parameters satisfying pre-defined constraints are retained from the prior set (Beven, 2006; Guillaume et al., 2015). It is worth repeating that even if the concept of a unique parameter vector is no longer used in parameter estimation, it is still useful in the context of identifiability analysis - in helping to rule out that non-uniqueness is due to sources I and II - which is often avoidable.

In addition to being non-unique, parameters estimated with noisy data may also be biased - in the sense that the mean parameter estimate may no longer represent the true value (where it is known). The uncertainty and bias in parameters also tend to decrease as sample size increases (depending on the properties of the errors), and increase as uncertainty in measurements increases.

These phenomena can be illustrated with our simple objective function $m(\theta)$, with a single (scalar) parameter. Consider the following model for oxygen consumption rate

$r=\frac{35}{8} \theta^{4} X_{1}-\frac{1}{80}(\theta-1)^{2} X_{2}+\frac{3}{8}$

where $\theta$ is again the biomass parameter. We take two noise-free measurements at $X_{1}=1, X_{2}=1$. We then investigate behaviour of the objective function for different true parameter values, summarised in Fig. 6. In the top row of the figure, we have set $\theta^{t}=0.5$ and the bottom row we have set $\theta^{t}=0.7$. In the first column of Fig. 6 we plot the rate $r$ as a function of the parameter. The black dots depict $r\left(\theta^{t}\right)$ and the blue dots represent one realization of noisy data $d=r\left(\theta^{t}\right)+\eta$, with $\eta=0.1$. In the second column of Fig. 6 we plot the loss functions $m(\theta)$ for noise- free and noisy observations. The loss function is minimized at the true parameter value (black dot). The blue dot represents the estimated parameter for the noisy data. The sensitivity of the recovered parameter is heavily dependent on the true parameter value, even for the same noise magnitude. In Fig. $6 \mathrm{~b}$, the parameter is less sensitive - the response surface is flatter with noisy data. Many parameters produce a rate $r$ that is close to the data. In contrast when $\theta^{t}=0.7$ (Fig. 6e), the model response has a much larger gradient around the estimated parameter.

A sampling procedure can be used to estimate the reliability of recovering the true parameter in the presence of noise of varying magnitude (Miao et al., 2011). For example, let the noise $\eta$ be normally distributed with mean zero and standard deviation $\sigma$. The third column of Fig. 6 depicts the recovered parameter values for 100 realizations of the noise, for three levels of noise $(\sigma \in\{0.01,0.05,0.1\})$. In every case, we find a unique minimum (the parameter is at least locally identifiable), but the minimum is no longer the true parameter that we could recover with noise-free data. The uncertainty of the estimated parameter depends on the noise level and the response surface in a local region around the true parameter. When the response surface is flat (top row), the parameter estimates are much more uncertain than when the response surface has a larger gradient (bottom row).

In practice, noise and errors are usually unavoidable. An analyst cannot tell for sure whether they are using or collecting the right type of information to obtain reliable estimates of parameters or model outputs representing real-world phenomena. Instead, they can only make an educated judgement that is dependent on how closely that model structure and data reflects reality. Identifiability analysis by definition tests for non-uniqueness with a given model structure and understanding of errors (and therefore objective function). If these assumptions poorly reflect reality, then identifiability analysis may be of limited use in informing the analyst's judgement. The better the model structure and the dataset, the more useful identifiability analysis will be. a)

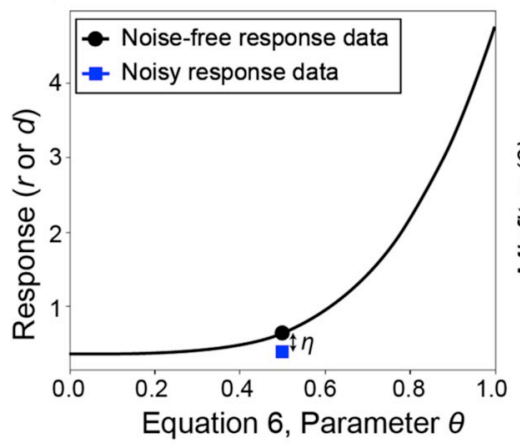

d)

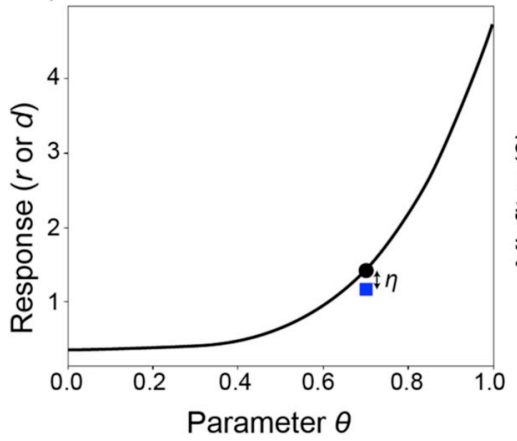

b)

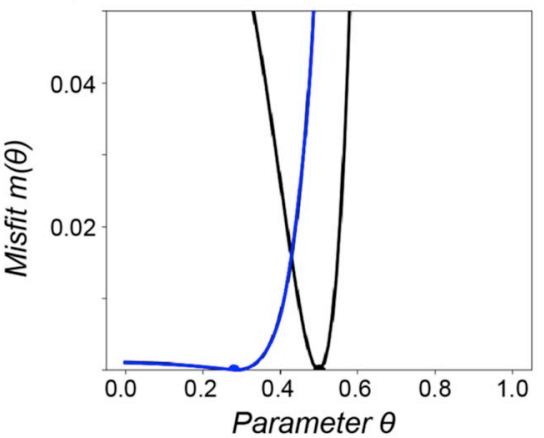

e)

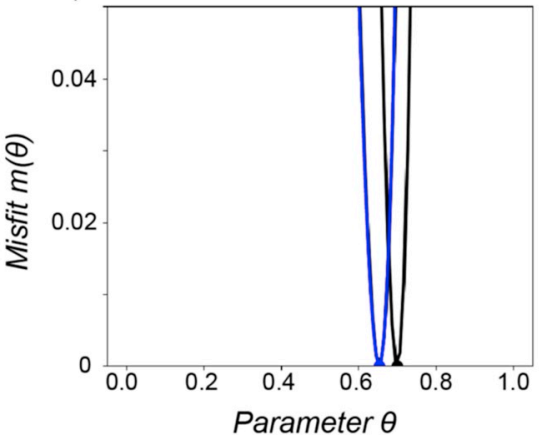

c)

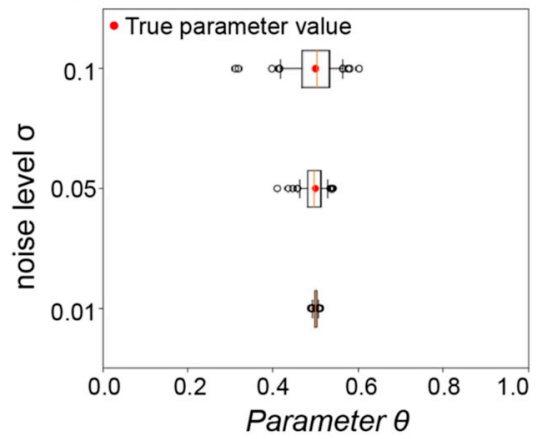

f)

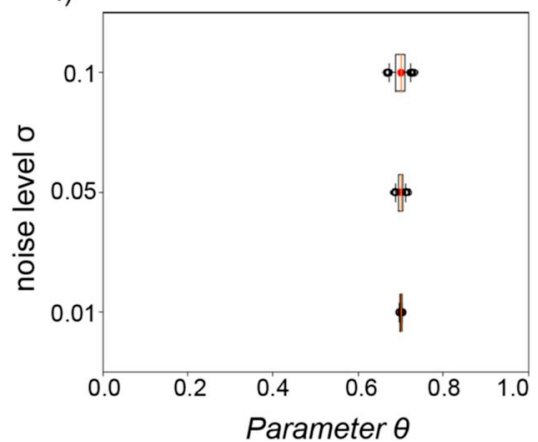

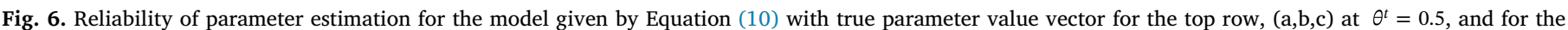

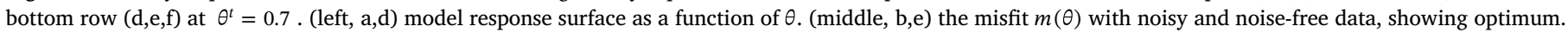
(right, c,f) the variability in the estimated parameter as a function of the noise level. $\sigma$ 


\section{Identifiability analysis in practice}

Parameter identifiability is a fundamental concept in parameter estimation, and therefore in many types of environmental modeling. Modelers should know whether the type of data they are using is capable of identifying unique parameter values. They should know if they do not have information about the right quantities within a given model structure (Source I). They should know if the forcing data (and associated response) does not reflect the conditions needed to estimate their parameters (Source II). And they should know if the errors are such that more data will not improve parameter uncertainty (Source III). First tests are often even trivial to implement: simply repeat an optimisation with different initialisations to check if it returns different values for parameters (see e.g. Shin et al., 2015). Sensitivity analysis can be performed using black box software, and dotty plots can easily be produced for each parameter, amongst other methods (Borgonovo et al., 2017).

Knowing that an identifiability problem exists, however, is only a starting point. Determining the cause of non-identifiability within a model structure, forcing data or messy dataset is likely to fall at one of two extremes: quite difficult, or trivial, for example, trying to estimate snow-related parameters on a tropical island. Excluding the trivial case, issues of identifiability tend by their very nature to be concealed from the modeler's view. If the issues were obvious, the modeler would have addressed them, and no identifiability issue would be experienced.

Similarly, improving identifiability is often non-trivial. It requires thorough understanding of the cause(s) of non-identifiability, and the time, knowledge and resources to collect or select new data, and modify model structures or objective functions. Even simple fixes such as fixing parameter values or using a different model structure may have significant, and often overlooked, impacts on results.

While tools are available to support identifiability analysis, there is still a great need for development of methods, software and training to ensure all modelers are able to assess and react appropriately to nonidentifiability. In the meantime, to provide some guidance, we make five pragmatic recommendations, and briefly discuss computational considerations.

\subsection{Know whether your model parameters are identifiable}

It cannot be repeated too often: identifiability is a fundamental concept in parameter estimation. Particularly given the difficulty of eliminating non-identifiability, it is important to explicitly acknowledge and document for other analysts how identifiability concerns have influenced the modeling process, and how they have been addressed. Regardless of the response taken, the analyst should be transparent about their treatment of identifiability. Documentation should describe whether the model is identifiable and how this was assessed. If the model is non-identifiable, the documentation should describe the expected consequences for the particular analysis, and the responses that were taken.

As a starting point, a modeler should keep an eye open for symptoms of non-identifiability (Box 1), and apply simple diagnostics like repeated optimisation, sensitivity analysis and 1 OR $2 \mathrm{D}$ dotty plots. If there are signs of non-identifiability, a reviewer should at least want to know what the source of the non-identifiability is in order to judge whether it is acceptable. Model structures of some complex models are known to be non-identifiable. For example, in spatially-distributed groundwater modeling, there is a common risk of interactions between parameters in adjacent grid cells, similar to the case described in equation (3). Uniquely estimating transmissivity therefore depends on having water level measurements within appropriate parts of the flow region (Neuman, 1973). Similar problems have also been observed for rainfall-runoff models with many parameters (Shin et al., 2015). If identifiability is not already known, techniques in Section 2 can be used either analytically, or with ideal input data and noise-free outputs, to show the model structure is responsible (Source I).

Using synthetic data (real input data and noise-free outputs) can reveal if the input data provide insufficient excitation of modes of model behaviour (Source II). For example, parameters of some hydrological models may only be active in rare conditions (Gupta and Sorooshian, 1983), such as observed streamflow in a drought period may not provide information about parameters related to flood periods, and vice-versa. For some models, it can be possible to use analytical methods to identify the data characteristics necessary to achieve persistence of excitation (Norton, 2009). Other techniques can also be used to assess information in data, such as by observing the effect of data on parameter sensitivity (Wagener et al., 2001). Examples include timevarying or spatially varying sensitivity analysis (Gupta and Razavi, 2018; Pianosi et al., 2016; Razavi and Gupta, 2019), and notablythe Dynamic Identifiability Analysis technique (Wagener et al., 2003).

If tests suggest that neither the structure (Source I), nor input data (Source II) are responsible, then the role of noise and systematic errors can be explored in input data, output data, model structure and error model, testing alternative model-data combinations. It may also be that the optimisation algorithm is failing to find the unique solution due to multiple regions of attraction, minor local optima, roughness, poor sensitivity and non-convex shape of the response surface (Duan et al., 1992).

\subsection{Consider how non-identifiability fits within your analysis}

In addition to helping to avoid negative effects of non-identifiability (Box 1), it is worth keeping in mind the benefits of identifiability analysis (Box 2) - there is a variety of ways it might influence modeling and model-based analyses in practice. While non-identifiability means the modeler lacks the appropriate information to choose between alternative models, in practice the right information may take too much time or resources to obtain, or may never be available at all. Depending on the prediction to be made, it may not matter that multiple models are plausible - the differences in prediction may be small enough to be acceptable, or able to be addressed by decision makers through adaptive management (Williams, 2011). How best to handle non-identifiability is highly dependent on purpose and context. The modeler must use their professional judgement to select practices that suit their problem (consistent with expectations in their modeling domain), and document the reasons for their choice, acknowledging possible

Box 1

Undesirable effects of non-identifiability

- Different runs/initialisations of an optimisation algorithms will return different values for parameters

- Using noise-free data still does not allow parameters (incl. initial conditions) to be uniquely identified

- Due to parameter non-uniqueness, estimates of physically meaningful parameters are not possible in models representing real-world processes

- It will not be possible to use the model to predict, with reasonable accuracy an unobserved, physically meaningful, model output

- Predictions may be inaccurate because the estimated parameters failed to capture key behaviours of the system that were not present during the model development period, such as due to climate change

- Estimates of insensitive parameters may be influenced by (overfitting) errors, or the modeler may have set them to an arbitrary value, such that the model gives good performance in model development for the wrong reasons 
Box 2

Benefits of identifiability analysis

- Assessing whether model complexity is suited to the type of information available in data

- Informing development of more parsimonious models, eliminating redundancy

- Identifying opportunities to reduce uncertainty and improve predictions

- Facilitating insight into the role of model structure assumptions, forcing data and errors on model output uncertainty

- Ensuring that the right type of information is used before embarking on further uncertainty analysis

- Using identifiability methods to understand and fix fundamental problems with model construction, making it easier to cope with other sources of uncertainty, such as in data (see e.g. La Vigna et al., 2016) or in coding

- Helping to plan what data needs to be collected

- Evaluating plans for data collection (e.g. Dausman et al., 2010)

- Understanding the estimation of parameters, before evaluating whether system states can be estimated and controlled (observability and controllability), which are also key topics in systems and control theory, outside the scope of this article (see Keesman, 2011)

- Establishing the behaviour of the model in known conditions, making it easier to understand behaviour of the model in broader conditions

alternatives.

In addition to simply tolerating non-identifiability, there are three other high level philosophical approaches possible (Wagener and Gupta, 2005), which can be viewed as: quantifying the effect of lack of information; improving the information used by obtaining the right type of observations and/or inputs; and avoiding the need for missing information by modifying the model. These four options are briefly described here.

Consider whether non-identifiability can be tolerated: Uncertainty due to identifiability issues (whether explicitly quantified or not) can be assessed in terms of its risk, that is, its effect on the final product of the analysis, such as quantities of predictive interest for decision making. For example, if the uncertainty induced by non-identifiability does not change a decision, then perhaps it can be ignored (Guillaume et al., 2015). This is typically the default approach if modelers are aware of identifiability issues. By professional judgment, modelers often assert that a given issue is not significant, in order to be able to provide results efficiently rather than futilely trying to tie up every loose end (Guillaume et al., 2017).

Consider quantifying uncertainty due to non-identifiability: It may be advantageous to formally quantify the effect of non-identifiable parameters. For example, if a process-based model is used to estimate model outputs not used in parameter estimation, then a complex model may be necessary even though it is not strictly identifiable. In the context of unsaturated zone models in hydrology, for example, Brunner et al. (2012) observed that certain observations can significantly reduce predictive uncertainty without informing any specific parameters. In cases such as these, the limitations of the model should be documented, and the uncertainty induced by non-identifiability should be quantified. For some problems, dedicated methods are available that identify parameter vectors that yield identical outputs or objective function values (e.g. Null-space Monte Carlo, Tonkin and Doherty, 2009).

Consider obtaining information to make the model identifiable: Some symptoms of identifiability may be considered intolerable, such as nonuniqueness of optimised parameters, lack of observability of a parameter, or lack of transferability of a model to specific conditions, for instance from flood to drought in hydrological applications. In these situations, steps can be taken to improve identifiability, by measuring different quantities (Source I), obtaining data in different conditions (Source II), or that is more accurate (Source III). Literature on data acquisition planning and optimal input design (e.g. Dausman et al., 2010; Freeze et al., 1992; Goodwin and Payne, 1977; Tiedeman et al., 2004; Walsh et al., 2017), along with using soft data and expert knowledge (Gharari et al., 2014) can be of assistance. Targeted reviews of types of data used for parameter estimation can also provide guidance (e.g. for hydrogeology, Schilling et al., 2019). Alternatively, it may be possible to constrain the solution space by making assumptions to reduce the number of unknowns, or providing information about which values are preferred using prior distributions (Stuart, 2010) or regularization (Tikhonov et al., 1995). Machine learning-based methods provide another example - non-uniqueness can occur due to redundant information in inputs (Maier et al., 2010) or local optima due to non-linearities (Kingston et al., 2005), and steps can be taken to avoid them, including through input variable selection (May et al., 2011).

Consider modifying model information requirements: It may be more appropriate to use a model that is identifiable with the available information (Grayson et al., 2002; Jakeman and Hornberger, 1993; Young et al., 1996). The principle of parsimony suggests that unnecessary complexity should be discarded - a number of model selection criteria prefer models with less parameters (e.g. Akaike Information Criterion,Akaike, 1974). In the case where particular observations can reduce predictive uncertainty without informing any specific parameters, information is likely being provided about combinations of parameters. These combinations could possibly be replaced by a single parameter within a simplified model (e.g. Croke and Jakeman, 2004; Young et al., 1996). This is different to eliminating parameters or fixing parameter values. A single combined parameter captures information from data about multiple, more detailed parameters and their total effect after accounting for interactions present in the parameter estimation data. Parameter elimination or fixing, on the other hand, introduces new information in the form of assumptions about the value of that parameter. If those assumptions are incorrect, even if the model performs well in some circumstances, it will be right for the wrong reasons, and may predict poorly.

Eliminating the symptoms of non-identifiability, however, typically requires invasive changes to the model or model identification procedure, for example using different or transformed parameters, selecting specific data periods, changing model structure, and/or using a more sophisticated objective function. It is important that these changes do not undermine the ability to understand the concepts that went into creating the model in the first place.

\subsection{Computational considerations}

Constructing response surfaces, cross sections, dotty plots, exploring parameter sensitivities or estimating the impact of noise can require large numbers of model evaluations. This can render identifiability analysis infeasible if the model being analysed is computationally expensive. While high performance computing may be a solution in some cases, it may also be possible to use computationally frugal methods (Hill et al., 2016), or to build an approximation of the model's response surface, to reduce the computational demands of identifiability analysis. The remainder of this section focuses on this last option, which is rapidly maturing.

A response surface can be explicitly represented mathematically by model emulation, resulting in a surrogate model. This has two key advantages. Firstly, having an analytical representation of the response surface allows additional mathematical analyses. Secondly, the computational cost of building and running a surrogate model is typically 
less than relying on the original model, therefore facilitating the use of more computationally intensive methods. The efficacy of response surface methods depends on the ability to build and capture the salient features of the response surface with a feasible number of model simulations. The approach known as Response Surface Methodology (Box and Draper, 1987) approximates the response surface by a quadratic function and allows analysis of flatness near the optimum. Although requiring a limited number of model simulations, quadratic response surfaces are often poor representations of a model response surface. Alternative methods that can approximate the non-linearity of the model response and higher-order interactions between variables are typically needed.

Polynomial chaos expansions (PCE) (Sudret, 2008; Xiu and Karniadakis, 2002) and Gaussian processes (GP) (Marrel et al., 2009; Oakley and O'Hagan, 2004) are two popular and effective methods for approximating highly non-linear response surfaces. GP and PCE can both be efficiently used as surrogates for the simulation model in any of the numerical identifiability methods outlined in this paper. For example, gradient and Hessian data can be computed analytically from the approximations and used for derivative-based identifiability analysis or the approximations can be used as surrogates within the likelihood function when conducting Bayesian inference (e.g. Blanchard et al., 2010) or calibration using maximum likelihood estimation (e.g. Arendt et al., 2012). In the water resources sector and in groundwater modeling particularly, Razavi et al. (2012) and Asher et al. (2015) respectively provide reviews of a range of surrogate modeling methods.

In addition to the aforementioned techniques based upon function approximation, reduced order models can also be used to decrease the computational cost of analyzing expensive simulation models (Carlberg, 2015; Cui et al., 2015; Soize and Farhat, 2017). These methods do not construct response surface approximations but rather solve the governing equations on a reduced basis. The advantage of such an approach is that one can cheaply obtain approximations of the entire solution to the governing equations rather than a small number of functions of that solution. Moreover, unlike surrogate methods, reduced order models do not suffer from the curse of parameter dimensionality.

The number of simulations required to build a response surface grows rapidly with parameter dimension. Consequently, the practical use of response surface methods is typically restricted to models with the order of 10 parameters. For example, a survey of the literature has shown that less than $65 \%$ of the applications of these methods are on functions having less than ten parameters, and more than $85 \%$ have less than 20 (Razavi et al., 2012). The exact number of simulations required to achieve a given accuracy is model dependent. However simulation requirements can be reduced by using methods that exploit the structure in the response surface (Constantine, 2015; Gorodetsky and Jakeman, 2018; Jakeman et al., 2015) and goal-oriented approximations, thereby focussing computational effort on regions and/or dimensions of parameter space that significantly affect quantities of interest. Lastly, when using a surrogate model, the user needs to be mindful of "approximation uncertainty", that is the potential errors of the surrogate model that can be added to the process of parameter estimation. Razavi et al. (2012) illustrate this source of uncertainty by simple examples (see Figs. 2, 3 and 6 therein) and review methods to address it.

\section{Conclusions}

This article has provided an introductory overview to key issues in identifiability analysis, and fundamental concepts and methods to quantify and understand the impact of non-uniqueness of parameters on modeling results. Identifiability analysis aims to (i) assess whether it is possible to identify unique parameter values, and (ii) understand why that occurs. It therefore makes an essential contribution to more systematically learning about sources of uncertainty and how to reduce them, and hence improving the practice, credibility and outcomes of modeling exercises in the long run.

Our discussion of identifiability analysis in practice emphasizes that the right tools to use depend on modeling context, especially the purpose, and the modeler's professional judgement. Our fundamental recommendation is therefore that assessment of whether a modeling exercise is fit for purpose explicitly needs to address three points:

- whether the model is identifiable

- the source(s) of any non-identifiability issue

- the extent to which any non-identifiability impacts the problem at hand.

It is important to explicitly acknowledge and document for other analysts how identifiability concerns have influenced the modeling process, and how they have been addressed. Regardless of the response taken, the analyst should be transparent about their treatment of identifiability. Documentation should describe whether the model is identifiable and how this was assessed. If the model is non-identifiable, the documentation should describe the expected consequences for the particular analysis, and the responses that were taken.

The modeler's professional judgement naturally depends on knowing what alternative methods of identifiability analysis are available, and why they might be useful. The emphasis of this introductory overview is therefore on awareness raising, encouraging wider acknowledgement of non-identifiability as a critical issue and that there are methods to assess it and its sources. There is, however, still much to be done to make practices for assessing identifiability easier to apply, and for them to be commonly applied. And there are huge opportunities to improve identifiability of models and reduce uncertainties, especially where there is a large community of resourceful researchers that uses a particular non-identifiable model. There are several well-known hydrological and water quality models, for instance, where sensitivity analysis indicates they are structurally non-identifiable. But other sectors are replete with over-parameterized models that do not use mitigating mechanisms (such as regularization) or investigate alternative model structure hypotheses that may lead to improved identifiability. Promoting the thoughtful use of identifiability analysis practices provides a firm foundation for improving models into the future.

\section{Acknowledgements}

This introductory overview builds on an iEMSs conference paper (Marsili-Libelli et al., 2014). Joseph Guillaume was supported by Academy of Finland funded project WASCO (grant no. 305471) and Emil Aaltonen Foundation funded project 'eat-less-water'.

The views expressed in the article do not necessarily represent the views of the U.S. Department of Energy or the United States Government. Sandia National Laboratories is a multimission laboratory managed and operated by National Technology and Engineering Solutions of Sandia, LLC., a wholly owned subsidiary of Honeywell International, Inc., for the U.S. Department of Energy's National Nuclear Security Administration under contract DE-NA-0003525.

\section{References}

Sobol, I., 2001. Global sensitivity indices for nonlinear mathematical models and their Monte Carlo estimates. Math. Comput. Simulat. 55, 271-280. https://doi.org/10. 1016/S0378-4754(00)00270-6.

Akaike, H., 1974. A new look at the statistical model identification. IEEE Trans. Autom. Control 19, 716-723. https://doi.org/10.1109/TAC.1974.1100705.

Arendt, P.D., Apley, D.W., Chen, W., Lamb, D., Gorsich, D., 2012. Improving identifiability in model calibration using multiple responses. J. Mech. Des. 134, 100909. https://doi.org/10.1115/1.4007573.

Asher, M.J., Croke, B.F.W., Jakeman, A.J., Peeters, L.J.M., 2015. A review of surrogate models and their application to groundwater modeling. Water Resour. Res. 51 n/a-n/ a. https://doi.org/10.1002/2015WR016967.

Badham, J., Elsawah, S., Guillaume, J.H.A., Hamilton, S.H., Hunt, R.J., Jakeman, A.J., 
Pierce, S.A., Snow, V.O., Babbar-Sebens, M., Fu, B., Gober, P., Hill, M.C., Iwanaga, T., Loucks, D.P., Merritt, W.S., Peckham, S.D., Richmond, A.K., Ames, D., Bammer, G., 2019. Effective modeling for Integrated Water Resource Management: a guide to contextual practices by phases and steps and future opportunities. Environ. Model. Softw 116, 40-56. https://doi.org/10.1016/j.envsoft.2019.02.013.

Balsa-Canto, E., Banga, J.R., 2011. AMIGO, a toolbox for advanced model identification in systems biology using global optimization. Bioinformatics 27, 2311-2313. https:// doi.org/10.1093/bioinformatics/btr370.

Beck, M.B., 1987. Water quality modeling: a review of the analysis of uncertainty. Water Resour. Res. 23, 1393-1442. https://doi.org/10.1029/WR023i008p01393.

Bellman, R., Åström, K.J., 1970. On structural identifiability. Math. Biosci. 7, 329-339. https://doi.org/10.1016/0025-5564(70)90132-X.

Bennett, N.D., Croke, B.F.W., Guariso, G., Guillaume, J.H.A., Hamilton, S.H., Jakeman, A.J., Marsili-Libelli, S., Newham, L.T.H., Norton, J.P., Perrin, C., Pierce, S.A., Robson, B., Seppelt, R., Voinov, A.A., Fath, B.D., Andreassian, V., 2013. Characterising performance of environmental models. Environ. Model. Softw 40,1-20. https://doi.org/ 10.1016/j.envsoft.2012.09.011.

Beven, K., 2006. A manifesto for the equifinality thesis. J. Hydrol. 320, 18-36. https:// doi.org/10.1016/j.jhydrol.2005.07.007.

Blanchard, E.D., Sandu, A., Sandu, C., 2010. Polynomial chaos-based parameter estimation methods applied to a vehicle system. Proc. Inst. Mech. Eng. - Part K J. Multi-body Dyn. 224, 59-81. https://doi.org/10.1243/14644193JMBD204.

Borgonovo, E., Lu, X., Plischke, E., Rakovec, O., Hill, M.C., 2017. Making the most out of a hydrological model data set: sensitivity analyses to open the model black-box. Water Resour. Res. 53, 7933-7950. https://doi.org/10.1002/2017WR020767.

Box, G.E.P., Draper, N.R., 1987. Empirical Model-Building and Response Surfaces. Wiley, New York.

Brun, R., Reichert, P., Künsch, H.R., 2001. Practical identifiability analysis of large environmental simulation models. Water Resour. Res. 37, 1015-1030. https://doi.org/ 10.1029/2000WR900350.

Brunner, P., Doherty, J., Simmons, C.T., 2012. Uncertainty assessment and implications for data acquisition in support of integrated hydrologic models. Water Resour. Res. 48. https://doi.org/10.1029/2011WR011342.

Campolongo, F., Cariboni, J., Saltelli, A., 2007. An effective screening design for sensitivity analysis of large models. Environ. Model. Softw 22, 1509-1518. https://doi. org/10.1016/j.envsoft.2006.10.004.

Carlberg, K., 2015. Adaptive h-refinement for reduced-order models. Int. J. Numer. Methods Eng. 102, 1192-1210. https://doi.org/10.1002/nme.4800.

Checchi, N., Giusti, E., Marsili-Libelli, S., 2007. PEAS: a toolbox to assess the accuracy of estimated parameters in environmental models. Environ. Model. Softw 22, 899-913. https://doi.org/10.1016/j.envsoft.2006.05.019.

Chiş, O., Banga, J.R., Balsa-Canto, E., 2011. GenSSI: a software toolbox for structura identifiability analysis of biological models. Bioinformatics 27 (18), 2610-2611. https://doi.org/10.1093/bioinformatics/btr431.

Clark, M.P., Kavetski, D., Fenicia, F., 2011. Pursuing the method of multiple working hypotheses for hydrological modeling. Water Resour. Res. 47. https://doi.org/10 1029/2010WR009827.

Cobelli, C., DiStefano 3rd, J.J., 1980. Parameter and structural identifiability concepts and ambiguities: a critical review and analysis. Am. J. Physiol. Regul. Integr. Comp Physiol. 239, R7-R24. https://doi.org/10.1152/ajpregu.1980.239.1.R7.

Constantine, P.G., 2015. Active Subspaces. Society for Industrial and Applied Mathematics, Philadelphia, PA. https://doi.org/10.1137/1.9781611973860.

Constantine, P.G., Dow, E., Wang, Q., 2014. Active subspace methods in theory and practice: applications to kriging surfaces. SIAM J. Sci. Comput. 36, A1500-A1524. https://doi.org/10.1137/130916138.

Croke, B., Jakeman, A., 2004. A catchment moisture deficit module for the IHACRES rainfall-runoff model. Environ. Model. Softw 19, 1-5. https://doi.org/10.1016/j. envsoft.2003.09.001.

Cui, T., Marzouk, Y.M., Willcox, K.E., 2015. Data-driven model reduction for the Bayesian solution of inverse problems. Int. J. Numer. Methods Eng. 102, 966-990. https://doi. org $110.1002 / n m e .4748$.

Dausman, A.M., Doherty, J., Langevin, C.D., Sukop, M.C., 2010. Quantifying data worth toward reducing predictive uncertainty. Gr. Water 48, 729-740. https://doi.org/10. 1111/j.1745-6584.2010.00679.x.

DiStefano III, J., Cobelli, C., 1980. On parameter and structural identifiability: Nonunique observability/reconstructibility for identifiable systems, other ambiguities, and new definitions. IEEE Trans. Autom. Control 25, 830-833. https://doi.org/10.1109/TAC. 1980.1102439.

Dobre, S., Bastogne, T., Profeta, C., Barberi-Heyob, M., Richard, A., 2012. Limits of variance-based sensitivity analysis for non-identifiability testing in high dimensional dynamic models. Automatica 48, 2740-2749. https://doi.org/10.1016/j.automatica. 2012.05.004.

Doherty, J., Hunt, R.J., 2009. Two statistics for evaluating parameter identifiability and error reduction. J. Hydrol. 366, 119-127. https://doi.org/10.1016/j.jhydrol.2008. 12.018.

Duan, Q., Sorooshian, S., Gupta, V., 1992. Effective and efficient global optimization for conceptual rainfall-runoff models. Water Resour. Res. 28, 1015-1031. https://doi. org/10.1029/91wr02985.

Efstratiadis, A., Koutsoyiannis, D., 2010. One decade of multi-objective calibration approaches in hydrological modelling: a review. Hydrol. Sci. J. 55, 58-78. https://doi. org/10.1080/02626660903526292.

Freeze, R.A., James, B., Massmann, J., Sperling, T., Smith, L., 1992. Hydrogeological decision analysis: 4 . The concept of data worth and its use in the development of site investigation strategies. Gr. Water 30, 574-588. https://doi.org/10.1111/j.17456584.1992.tb01534.x.

Gharari, S., Hrachowitz, M., Fenicia, F., Gao, H., Savenije, H.H.G., 2014. Using expert knowledge to increase realism in environmental system models can dramatically reduce the need for calibration. Hydrol. Earth Syst. Sci. 18, 4839-4859. https://doi. org/10.5194/hess-18-4839-2014.

Godfrey, K.R., DiStefano III, J.J., 1987. Chapter 1 - IDENTIFIABILITY OF MODEL PARAMETERS. In: Walter, E. (Ed.), Identifiability of Parametric Models. Pergamon, pp. 1-20. https://doi.org/10.1016/B978-0-08-034929-9.50005-4.

Goodwin, G., Payne, R., 1977. Dynamic System Identification. Experiment Design and Data Analysis, Mathematics in Science and Engineering. Academic Press.

Gorodetsky, A.A., Jakeman, J.D., 2018. Gradient-based Optimization for Regression in the Functional Tensor-Train Format. arXiv [Preprint] arXiv:1801.00885.

Grayson, R.B., Blöschl, G., Western, A.W., McMahon, T.A., 2002. Advances in the use of observed spatial patterns of catchment hydrological response. Adv. Water Resour. 25, 1313-1334. https://doi.org/10.1016/S0309-1708(02)00060-X.

Guillaume, J.H.A., Kummu, M., Räsänen, T.A., Jakeman, A.J., 2015. Prediction under uncertainty as a boundary problem: a general formulation using Iterative Closed Question Modelling. Environ. Model. Softw 70, 97-112. https://doi.org/10.1016/j. envsoft.2015.04.004.

Guillaume, J.H.A., Helgeson, C., Elsawah, S., Jakeman, A.J., Kummu, M., 2017. Toward Best Practice Framing of Uncertainty in Scientific Publications: A Review of Water Resources Research Abstracts. Water Resour. Res. 53 (8), 6744-6762. https://doi. org/10.1002/2017WR020609.

Gupta, H.V., Razavi, S., 2018. Revisiting the basis of sensitivity analysis for dynamical earth system models. Water Resour. Res. 54, 8692-8717. https://doi.org/10.1029/ 2018WR022668.

Gupta, V.K., Sorooshian, S., 1983. Uniqueness and observability of conceptual rainfallrunoff model parameters: the percolation process examined. Water Resour. Res. 19, 269-276. https://doi.org/10.1029/WR019i001p00269.

Gupta, H.V., Sorooshian, S., Yapo, P.O., 1998. Toward improved calibration of hydrologic models: multiple and noncommensurable measures of information. Water Resour. Res. 34, 751-763. https://doi.org/10.1029/97WR03495.

Gupta, H.V., Clark, M.P., Vrugt, J.A., Abramowitz, G., Ye, M., 2012. Towards a comprehensive assessment of model structural adequacy. Water Resour. Res. 48. https:// doi.org/10.1029/2011WR011044.

Haghnegahdar, A., Razavi, S., 2017. Insights into sensitivity analysis of Earth and environmental systems models: on the impact of parameter perturbation scale. Environ. Model. Softw 95, 115-131. https://doi.org/10.1016/j.envsoft.2017.03.031.

Hill, M.C., Tiedeman, C.R., 2007. Effective Groundwater Model Calibration : with Analysis of Data, Sensitivities, Predictions, and Uncertainty. Wiley-Interscience.

Hill, M.C., Kavetski, D., Clark, M., Ye, M., Arabi, M., Lu, D., Foglia, L., Mehl, S., 2016 Practical use of computationally frugal model analysis methods. Gr. Water 54 159-170. https://doi.org/10.1111/gwat.12330.

Homma, T., Saltelli, A., 1996. Importance measures in global sensitivity analysis of nonlinear models. Reliab. Eng. Syst. Saf. 52, 1-17. https://doi.org/10.1016/0951 8320(96)00002-6.

Jakeman, A.J., Hornberger, G.M., 1993. How much complexity is warranted in a rainfallrunoff model? Water Resour. Res. 29, 2637-2649. https://doi.org/10.1029/ 93WR00877.

Jakeman, A.J., Letcher, R.A., Norton, J.P., 2006. Ten iterative steps in development and evaluation of environmental models. Environ. Model. Softw 21, 602-614. https:// doi.org/10.1016/j.envsoft.2006.01.004.

Jakeman, J.D., Eldred, M.S., Sargsyan, K., 2015. Enhancing $\ell 1$-minimization estimates of polynomial chaos expansions using basis selection. J. Comput. Phys. 289, 18-34. https://doi.org/10.1016/j.jcp.2015.02.025.

Karlsson, J., Anguelova, M., Jirstrand, M., 2012. An efficient method for structural identifiability analysis of large dynamic systems*. IFAC Proc 45, 941-946. https:// doi.org/10.3182/20120711-3-BE-2027.00381.

Keesman, K.J., 2011. System Identification, Advanced Textbooks in Control and Signal Processing. Springer London, London. https://doi.org/10.1007/978-0-85729-522-4.

Keesman, K.J., Doeswijk, T.G., 2009. Direct least-squares estimation and prediction of rational systems: application to food storage. J. Process Control 19, 340-348. https:// doi.org/10.1016/j.jprocont.2008.03.006.

Kelly (Letcher), R.A., Jakeman, A.J., Barreteau, O., Borsuk, M.E., ElSawah, S., Hamilton, S.H., Henriksen, H.J., Kuikka, S., Maier, H.R., Rizzoli, A.E., van Delden, H., Voinov, A.A., 2013. Selecting among five common modelling approaches for integrated environmental assessment and management. Environ. Model. Softw 47, 159-181. https://doi.org/10.1016/j.envsoft.2013.05.005.

Kingston, G.B., Maier, H.R., Lambert, M.F., 2005. Calibration and validation of neura networks to ensure physically plausible hydrological modeling. J. Hydrol. 314, 158-176. https://doi.org/10.1016/j.jhydrol.2005.03.013.

Koopmans, T.C., Reiersol, O., 1950. The identification of structural characteristics. Ann. Math. Stat. 21, 165-181.

La Vigna, F., Hill, M.C., Rossetto, R., Mazza, R., 2016. Parameterization, sensitivity analysis, and inversion: an investigation using groundwater modeling of the surfacemined Tivoli-Guidonia basin (Metropolitan City of Rome, Italy). Hydrogeol. J. 24, 1423-1441. https://doi.org/10.1007/s10040-016-1393-z.

Ljung, L., Glad, T., 1994. On global identifiability for arbitrary model parametrizations. Automatica 30, 265-276. https://doi.org/10.1016/0005-1098(94)90029-9.

Maier, H.R., Jain, A., Dandy, G.C., Sudheer, K.P., 2010. Methods used for the development of neural networks for the prediction of water resource variables in river systems: Current status and future directions. Environ. Model. Softw 25, 891-909. https://doi.org/10.1016/j.envsoft.2010.02.003.

Maier, H.R., Kapelan, Z., Kasprzyk, J., Kollat, J., Matott, L.S., Cunha, M.C., Dandy, G.C., Gibbs, M.S., Keedwell, E., Marchi, A., Ostfeld, A., Savic, D., Solomatine, D.P., Vrugt, J.A., Zecchin, A.C., Minsker, B.S., Barbour, E.J., Kuczera, G., Pasha, F., Castelletti, A., Giuliani, M., Reed, P.M., 2014. Evolutionary algorithms and other metaheuristics in water resources: Current status, research challenges and future directions. Environ. 
Model. Softw 62, 271-299. https://doi.org/10.1016/j.envsoft.2014.09.013.

Maier, H.R., Razavi, S., Kapelan, Z., Matott, L.S., Kasprzyk, J., Tolson, B.A., 2019. Introductory overview: optimization using evolutionary algorithms and other metaheuristics. Environ. Model. Softw 114, 195-213. https://doi.org/10.1016/j.envsoft 2018.11.018.

Marrel, A., Iooss, B., Laurent, B., Roustant, O., 2009. Calculations of Sobol indices for the Gaussian process metamodel. Reliab. Eng. Syst. Saf. 94, 742-751. https://doi.org/10. 1016/j.ress.2008.07.008.

Marsili-Libelli, S., 1992. Parameter estimation of ecological models. Ecol. Model. 62, 233-258. https://doi.org/10.1016/0304-3800(92)90001-U.

Marsili-Libelli, S., 2016. Environmental Systems Analysis with MATLAB. CRC Press.

Marsili-Libelli, S., Guerrizio, S., Checchi, N., 2003. Confidence regions of estimated parameters for ecological systems. Ecol. Model. 165, 127-146. https://doi.org/10. 1016/S0304-3800(03)00068-1.

Marsili-Libelli, S., Beck, M.B., Brunner, P., Croke, B., Guillaume, J., Jakeman, A., Jakeman, J., Keesman, K., Stigter, H., 2014. Practical identifiability analysis of environmental models. In: Proceedings - 7th International Congress on Environmental Modelling and Software: Bold Visions for Environmental Modeling, IEMSs 2014.

Matott, L.S., Babendreier, J.E., Purucker, S.T., 2009. Evaluating uncertainty in integrated environmental models: a review of concepts and tools. Water Resour. Res. 45. https://doi.org/10.1029/2008WR007301.

May, R., Dandy, G., Maier, H., 2011. Review of input variable selection methods for artificial neural networks. In: Artificial Neural Networks - Methodological Advances and Biomedical Applications. InTech. https://doi.org/10.5772/16004.

Miao, H., Xia, X., Perelson, A.S., Wu, H., 2011. On identifiability of nonlinear ODE models and applications in viral dynamics. SIAM Rev. 53, 3-39. https://doi.org/10.1137/ 090757009.

Milly, P.C.D., Betancourt, J., Falkenmark, M., Hirsch, R.M., Kundzewicz, Z.W., Lettenmaier, D.P., Stouffer, R.J., 2008. Stationarity is dead: whither water management? Science 319, 573-574. (80- ). https://doi.org/10.1126/science 1151915.

Nearing, G.S., Gupta, H.V., 2015. The quantity and quality of information in hydrologic models. Water Resour. Res. 51, 524-538. https://doi.org/10.1002/2014WR015895.

Neuman, S.P., 1973. Calibration of distributed parameter groundwater flow models viewed as a multiple-objective decision process under uncertainty. Water Resour. Res. 9, 1006-1021. https://doi.org/10.1029/WR009i004p01006.

Norton, J.P., 1980. Normal-mode identifiability analysis of linear compartmental systems in linear stages. Math. Biosci. 50, 95-115. https://doi.org/10.1016/0025-5564(80) 90124-8.

Norton, J.P., 1982. An investigation of the sources of nonuniqueness in deterministic identifiability. Math. Biosci. 60, 89-108. https://doi.org/10.1016/0025-5564(82) 90033-5.

Norton, J.P., 2009. An Introduction to Identification. Dover Publications, Inc., New York, NY, USA.

Norton, J., 2015. An introduction to sensitivity assessment of simulation models. Environ. Model. Softw 69, 166-174. https://doi.org/10.1016/j.envsoft.2015.03.020.

Norton, J.P., Brown, R.F., Godfrey, K.R., 1980. Modal analysis of identifiablity of linear compartmental models. IEE Proc. D Control Theory Appl. 127, 83-92. https://doi. org/10.1049/ip-d.1980.0014.

Oakley, J.E., O'Hagan, A., 2004. Probabilistic sensitivity analysis of complex models: a Bayesian approach. J. R. Stat. Ser. Soc. B Stat. Methodol. 66, 751-769. https://doi. org/10.1111/j.1467-9868.2004.05304.x.

Pianosi, F., Beven, K., Freer, J., Hall, J.W., Rougier, J., Stephenson, D.B., Wagener, T., 2016. Sensitivity analysis of environmental models: a systematic review with prac tical workflow. Environ. Model. Softw 79, 214-232. https://doi.org/10.1016/j. envsoft.2016.02.008.

Rakovec, O., Hill, M.C., Clark, M.P., Weerts, A.H., Teuling, A.J., Uijlenhoet, R., 2014. Distributed evaluation of local sensitivity analysis (DELSA), with application to hydrologic models. Water Resour. Res. 50, 409-426. https://doi.org/10.1002/ 2013 WR014063.

Raue, A., Kreutz, C., Maiwald, T., Bachmann, J., Schilling, M., Klingmüller, U., Timmer, J., 2009. Structural and practical identifiability analysis of partially observed dynamical models by exploiting the profile likelihood. Bioinformatics 25, 1923-1929. https://doi.org/10.1093/bioinformatics/btp358.

Razavi, S., Gupta, H.V., 2015. What do we mean by sensitivity analysis? The need for comprehensive characterization of "global" sensitivity in Earth and Environmental systems models. Water Resour. Res. 51, 3070-3092. https://doi.org/10.1002/ 2014 WR016527.

Razavi, S., Gupta, H.V., 2016a. A new framework for comprehensive, robust, and efficient global sensitivity analysis: 1 . Theory. Water Resour. Res. 52, 423-439. https://doi. org/10.1002/2015WR017558.

Razavi, S., Gupta, H.V., 2016b. A new framework for comprehensive, robust, and efficient global sensitivity analysis: 2. Application. Water Resour. Res. 52, 440-455. https:// doi.org/10.1002/2015WR017559.

Razavi, S., Gupta, H.V., 2019. A multi-method Generalized Global Sensitivity Matrix approach to accounting for the dynamical nature of earth and environmental systems models. Environ. Model. Softw 114, 1-11. https://doi.org/10.1016/j.envsoft.2018. 12.002

Razavi, S., Tolson, B.A., Burn, D.H., 2012. Review of surrogate modeling in water resources. Water Resour. Res. 48. https://doi.org/10.1029/2011WR011527.

Razavi, S., Sheikholeslami, R., Gupta, H.V., Haghnegahdar, A., 2019. VARS-TOOL: toolbox for comprehensive, efficient, and robust sensitivity and uncertainty analysis. Environ. Model. Softw 112, 95-107. https://doi.org/10.1016/j.envsoft.2018.10.005.

Refsgaard, J.C., van der Sluijs, J.P., Højberg, A.L., Vanrolleghem, P.A., 2007. Uncertainty in the environmental modelling process - a framework and guidance. Environ. Model. Softw 22, 1543-1556. https://doi.org/10.1016/j.envsoft.2007.02.004.

Rothenberg, T.J., 1971. Identification in parametric models. Econometrica 39, 577-591. https://doi.org/10.2307/1913267.

Saccomani, M.P., Bellu, G., 2008. DAISY: an efficient tool to test global identifiability. Some case studies. In: 2008 16th Mediterranean Conference on Control and Automation. IEEE, pp. 1723-1728. https://doi.org/10.1109/MED.2008.4602152.

Saltelli, A., Annoni, P., 2010. How to avoid a perfunctory sensitivity analysis. Environ. Model. Softw 25, 1508-1517. https://doi.org/10.1016/j.envsoft.2010.04.012.

Saltelli, A., Chan, K., Scott, E.M., 2000. Sensitivity Analysis Wiley Series in Probability and Statistics. Willey, New York.

Schilling, O.S., Cook, P.G., Brunner, P., 2019. Beyond classical observations in hydrogeology: the advantages of including exchange flux, temperature, tracer concentration, residence time, and soil moisture observations in groundwater model calibration. Rev. Geophys. 57, 146-182. https://doi.org/10.1029/2018RG000619.

Schoups, G., Vrugt, J.A., 2010. A formal likelihood function for parameter and predictive inference of hydrologic models with correlated, heteroscedastic, and non-Gaussian errors. Water Resour. Res. 46, n. https://doi.org/10.1029/2009WR008933 n/a-n/a

Seber, G.A.F., Wild, C.J., 1989. Nonlinear Regression, Wiley Series in Probability and Statistics. John Wiley \& Sons, Inc., Hoboken, NJ, USA. https://doi.org/10.1002/ 0471725315.

Shin, M.-J., Guillaume, J.H.A., Croke, B.F.W., Jakeman, A.J., 2015. A review of foundational methods for checking the structural identifiability of models: results for rainfall-runoff. J. Hydrol. 520, 1-16. https://doi.org/10.1016/j.jhydrol.2014.11.040.

Sobol, I.M., Kucherenko, S., 2009. Derivative based global sensitivity measures and their link with global sensitivity indices. Math. Comput. Simulat. 79, 3009-3017. https:// doi.org/10.1016/j.matcom.2009.01.023.

Soize, C., Farhat, C., 2017. A nonparametric probabilistic approach for quantifying uncertainties in low-dimensional and high-dimensional nonlinear models. Int. J. Numer Methods Eng. 109, 837-888. https://doi.org/10.1002/nme.5312.

Sorooshian, S., Gupta, V.K., 1985. The analysis of structural identifiability: theory and application to conceptual rainfall-runoff models. Water Resour. Res. 21, 487-495. https://doi.org/10.1029/WR021i004p00487.

Stigter, J.D., Molenaar, J., 2015. A fast algorithm to assess local structural identifiability. Automatica 58, 118-124. https://doi.org/10.1016/j.automatica.2015.05.004.

Stigter, J.D., Beck, M.B., Molenaar, J., 2017. Assessing local structural identifiability for environmental models. Environ. Model. Softw 93, 398-408. https://doi.org/10. 1016/j.envsoft.2017.03.006.

Stuart, A.M., 2010. Inverse problems: a Bayesian perspective. Acta Numer. 19, 451-559. https://doi.org/10.1017/S0962492910000061.

Sudret, B., 2008. Global sensitivity analysis using polynomial chaos expansions. Reliab. Eng. Syst. Saf. 93, 964-979. https://doi.org/10.1016/j.ress.2007.04.002.

Thomson, G.H., 1919. The proof or disproof of the existence of general ability. Br. J. Psychol. 9, 321-336. https://doi.org/10.1111/j.2044-8295.1919.tb00230.x 19041920

Thyer, M., Renard, B., Kavetski, D., Kuczera, G., Franks, S.W., Srikanthan, S., 2009. Critical evaluation of parameter consistency and predictive uncertainty in hydrological modeling: a case study using Bayesian total error analysis. Water Resour. Res. 45. https://doi.org/10.1029/2008WR006825.

Tiedeman, C.R., Ely, D.M., Hill, M.C., O'Brien, G.M., 2004. A method for evaluating the importance of system state observations to model predictions, with application to the Death Valley regional groundwater flow system. Water Resour. Res. 40, 1-14. https://doi.org/10.1029/2004WR003313.

Tikhonov, A.N., Goncharsky, A.V., Stepanov, V.V., Yagola, A.G., 1995. Numerical Methods for the Solution of Ill-Posed Problems. Springer Netherlands, Dordrecht. https://doi.org/10.1007/978-94-015-8480-7.

Tonkin, M., Doherty, J., 2009. Calibration-constrained Monte Carlo analysis of highly parameterized models using subspace techniques. Water Resour. Res. 45. https: doi.org/10.1029/2007WR006678.

Vanrolleghem, P.A., Keesman, K.J., 1996. Identification of biodegradation models under model and data uncertainty. Water Sci. Technol. 33. https://doi.org/10.1016/0273 1223(96)00192-8.

Vrugt, J.A., 2016. Markov chain Monte Carlo simulation using the DREAM software package: theory, concepts, and MATLAB implementation. Environ. Model. Softw 75, 273-316. https://doi.org/10.1016/j.envsoft.2015.08.013.

Wagener, T., Boyle, D.P., Lees, M.J., Wheater, H.S., Gupta, H.V., Sorooshian, S., 2001. A framework for development and application of hydrological models. Hydrol. Earth Syst. Sci. 5 (1), 13-26. http://dx.doi.org/10.5194/hess-5-13-2001.

Wagener, T., Gupta, H.V., 2005. Model identification for hydrological forecasting under uncertainty. Stoch. Environ. Res. Risk Assess. 19, 378-387. https://doi.org/10.1007/ s00477-005-0006-5.

Wagener, T., McIntyre, N., Lees, M.J., Wheater, H.S., Gupta, H.V., 2003. Towards reduced uncertainty in conceptual rainfall-runoff modelling: dynamic identifiability analysis. Hydrol. Process. 17, 455-476. https://doi.org/10.1002/hyp.1135.

Walsh, S.N., Wildey, T.M., Jakeman, J.D., 2017. Optimal experimental design using a consistent bayesian approach. ASCE-ASME J. Risk Uncertain. Eng. Syst. Part B Mech. Eng. 4, 011005. https://doi.org/10.1115/1.4037457.

Walter, E., Pronzato, L., 1996. On the identifiability and distinguishability of nonlinear parametric models. Math. Comput. Simulat. 42, 125-134. https://doi.org/10.1016/ 0378-4754(95)00123-9.

Williams, B.K., 2011. Passive and active adaptive management: approaches and an example. J. Environ. Manag. 92, 1371-1378. https://doi.org/10.1016/j.jenvman.2010. 10.039.

Xiu, D., Karniadakis, G.E., 2002. The wiener-askey polynomial chaos for stochastic differential equations. SIAM J. Sci. Comput. 24, 619-644. https://doi.org/10.1137/ S1064827501387826.

Young, P., Parkinson, S., Lees, M., 1996. Simplicity out of complexity in environmental modelling: occam's razor revisited. J. Appl. Stat. 23, 165-210. https://doi.org/10. $1080 / 02664769624206$. 